\title{
EEG source localization using a sparsity prior based on Brodmann areas
}

Sajib Saha ${ }^{1,7}$, Yakov Nesterets ${ }^{2,3}$, Rajib Rana ${ }^{4}$, Murat Tahtali ${ }^{1}$, Frank de Hoog $^{5}$ and Timur Gureyev ${ }^{3,5,6}$

1) University of New South Wales, Canberra, Australia

2) CSIRO Manufacturing, Melbourne, Australia

3) University of New England, Armidale, Australia

4) University of Southern Queensland, QLD, Australia<smiles>C1CCC1</smiles>

5) CSIRO Data61, Canberra, ACT, Australia

6) ARC Centre of Excellence for Advanced Molecular Imaging, School of Physics, The University of

Melbourne, Parkville, VIC, Australia

7) CSIRO Health and Biosecurity, Perth, WA, Australia

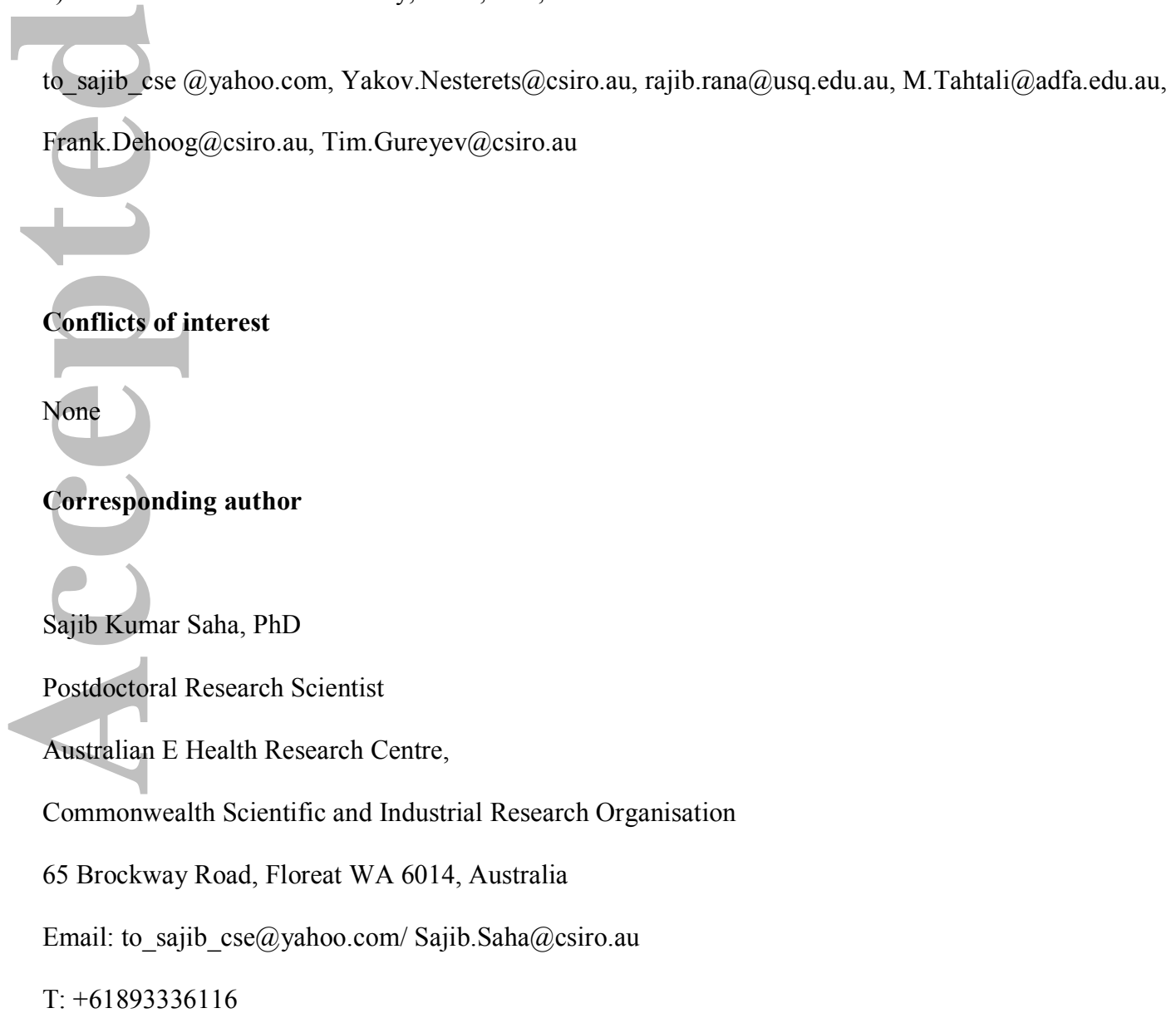

This is the author manuscript accepted for publication and has undergone full peer review but has not been through the copyediting, typesetting, pagination and proofreading process, which may lead to differences between this version and the Version record. Please cite this article as doi:10.1002/ ima.22236. 


\title{
EEG source localization using a sparsity prior based on Brodmann areas
}

\author{
Abstract \\ Localizing the sources of electrical activity in the brain from Electroencephalographic (EEG) data is an \\ important tool for non-invasive study of brain dynamics. Generally, the source localization process involves a \\ high-dimensional inverse problem that has an infinite number of solutions and thus requires additional \\ constraints to be considered to have a unique solution. In this paper we propose a novel method for EEG source \\ localization. The proposed method is based on dividing the cerebral cortex of the brain into a finite number of \\ "Functional Zones" which correspond to unitary functional areas in the brain. To specify the sparsity profile of \\ human brain activity more concisely, the proposed approach considers grouping of the electrical current dipoles \\ inside each of the Functional Zones. In this paper, we investigate the use of Brodmann's areas as the Functional \\ Zones while Sparse Bayesian Learning is used to perform sparse approximation. Numerical experiments are \\ conducted on a realistic head model obtained from segmentation of MRI images of the head and includes four \\ major compartments namely scalp, skull, cerebrospinal fluid (CSF) and brain with relative conductivity values. \\ Cor compr \\ Three different electrode setups are tested in the numerical experiments. The results demonstrate that the \\ proposed approach is quite promising in solving the EEG source localization problem. In a noiseless \\ environment with 71-electrodes, the proposed method was found to accurately locate up to 6 simultaneously \\ active sources with accuracy greater than $70 \%$.
}

Keywords Electroencephalography, source localization, inverse problem, Brodmann map, sparse

reconstruction

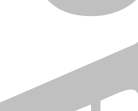

\section{CONFLICT OF INTEREST AND HUMAN SUBJECTS}

No conflict of interest to mention. No human or animal subjects were used for the experiment. 


\section{Introduction}

Understanding electrical activity inside the human $\operatorname{brain}^{1}$ is potentially of great diagnostic value for epilepsy $y^{2,3,4}$, stroke $^{5,6}$, traumatic brain injury ${ }^{7}$ and other brain disorders. Locating the sources of electrical activity inside the brain works by first finding the scalp potentials produced by virtual electric current dipoles at arbitrary locations

in the brain (i.e. solving the forward problem), then, in conjunction with the actual EEG data measured by the electrodes, it is used to work back and estimate the sources that best fit the measurements (i.e. solving the corresponding inverse problem). In the cases where the number of measurement points (i.e. electrodes, usually less than a $100^{8}$ ) is lower than the number of unknowns (i.e. potential positions inside the head of the electrical dipoles with unknown current strength and orientation, >1000) this inverse problem is severely ill-posed ${ }^{8}$ in the sense that there is an infinite number of source configurations that can produce the same distribution of the electric potential on the surface of the head. Hence, additional constraints need to be introduced in order to find an appropriate unique solution. Noting however that even with infinitely many data measurement points on the scalp, the spatial resolution of the EEG inversion will be limited due to the spreading of the electromagnetic signal on propagation through the head ${ }^{9}$.

Various methods have been proposed for choosing suitable constraints for the inverse EEG problem, the most well-known being the Minimum Norm $(\mathrm{MN})$ constraint ${ }^{10,11,12}$. Techniques relying on the MN constraint are based on a search for the solution with minimum power, along with regularization ${ }^{8}$. In other words, when the system is underdetermined, the solution is obtained by minimizing the $l_{2}$-norm of the solution components ${ }^{13}$. Several variants of this approach that consider different regularization parameters and weighting factors have already been proposed in the literature ${ }^{8,14}$. Among them Standardized LOw REsolution brain Electromagnetic Tomography (sLORETA) ${ }^{15}$ has gained significant attention because of its capacity to ensure zero localization error at least in the case of a single source and noiseless environment. Despite its accuracy in the case of a single source and the relative simplicity of the corresponding computations which can produce the results very fast, sLORETA has been criticized for generating very broadly distributed or "smeared" sources in the reconstruction region $^{16}$ and for poor performance in the case of multiple simultaneously activated sources ${ }^{17,18}$.

In the last two decades, significant efforts have been made to develop new improved methods for solving illposed problems using sparse priors. The FOCal Underdetermined System Solver (FOCUSS) is a classic example belonging to this category, which uses a weighted MN approach for sequentially reinforcing strong 
sources and suppressing the weak ones ${ }^{19}$. Other interesting algorithms are based on Iterative Reweighted LeastSquares (IRLS) methods, which are similar to FOCUSS and are based on iteratively computing weighted MN solutions with weights updated after each iteration ${ }^{20,21}$. The homotopy method by Osborne ${ }^{22}$ and the LARSLASSO algorithm $^{23,24}$ (a variant of the homotopy method) are extremely powerful methods for solving the $l_{1}$ problem. Simple coordinate descent methods ${ }^{25}$ or block wise coordinate descent, also called block coordinate relaxation $^{26}$, are also very successful strategies.

Following the discovery by Donoho and Candes et al. ${ }^{27,28}$ that sparsity could enable exact solution of ill-posed problems under certain conditions, there has been a tremendous growth of publications on efficient application of sparsity constraints for ill-posed problems ${ }^{29,30,31,32,33}$. In EEG, researchers have used sparseness constraints in spatial, spatiotemporal and frequency domains to reflect the focal nature of the cortical activity. Ding et al. in ${ }^{29,30}$ have proposed an $l_{1}$-norm electromagnetic source imaging method by exploring sparseness in a transform domain. To efficiently incorporate the sparsity prior, Wei-Tang et al. ${ }^{31}$ have considered Laplacian and Spherical wavelet transforms to solve the localization problem in MEG (magnetoencephalography). An interesting study about the performance of several state-of-the-art sparse approximation algorithms for solving EEG source localization problem was conducted by Zhang $\mathrm{in}^{32}$. Wu et al. $\mathrm{in}^{33}$ have proposed a matching pursuit based solution to the EEG inverse problem. While it produces better localization compared to the state-of-the art methods, the number of sources needs to be known a priori for the refinement of the localization in this method.

Despite the growing interest, the applicability of sparsity-enforcing priors for EEG source localization is still limited because of the significantly smaller number of electrodes compared to the typical number of virtual electric current dipoles in consideration. The algorithm by Wu et al. is the best to our knowledge and has only been able to accurately locate up to 6-8 active dipoles based on a 61-electrodes setup and considering only 1279

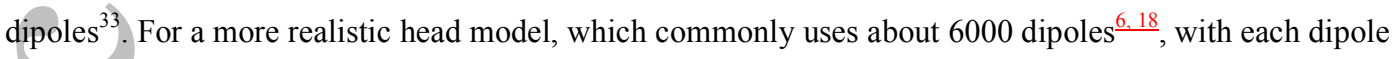
corresponding to about $5 \times 5 \times 5 \mathrm{~mm}^{3}$ of grey matter, this method is expected to give worse results for a similar electrode setup, because of the increased number of unknowns. Importantly, it is well accepted in the literature that a region of the brain corresponding to a group of virtual dipoles, rather than to a single dipole, tends to be activated during a certain brain activity ${ }^{9}$. For example, it is well known that Brodmann area 17 is related to human visual activity. In a realistic model ${ }^{18}$ with 6203 dipoles, the number of dipoles that belong to that region is about 54 . Now, if a group of more than 8 dipoles in that area are activated simultaneously, Therefore, even the best sparsity-based reconstruction method proposed so far will likely fail ${ }_{2}$ as it is far beyond the number of 
active dipoles that the method can handleto locate the activity accurately, if a group of more than 8 dipeles in that area are activated simultaneously. It has been pointed out by Wagner and colleagues ${ }^{17}$ that in the sLORETA method, closely located activated dipoles will produce a broad region having activity maxima located somewhere in between, therefore sLORETA may produce acceptable results in such a scenario. At the same time, sLORETA will likely produce inaccurate results when more than one group of dipoles corresponding to different spatially separated areas of the brain are activated simultaneously. In this perspective, relying on Assuming typical activation of the group of dipoles rather than a single dipole and considering the severely underdetermined nature of the problem, the sparsity-based reconstruction methodology proposed in this paper groups the dipoles based on their activity, and then applies sparsity constraint for detection of activated groups or region(s) in the brain. It is worth mentioning that Friston $e t a l^{34}$ has found such dimensionality reduction as an efficient strategy to solve the EEG/MEG inverse problem. The authors have proposed inverse solution scheme for the EEG/MEG inverse problem, which considers a model reduction by projection onto a spatiotemporal subspace, followed by variational inversion of the ensuring hierarchical or parametric empirical Bayes model.

(C.

In this work, we aim to reduce the dimensionality of the inverse problem based on some heuristic of grouping the dipoles. Since the full functionality of different parts of the human brain is still not completely known, the grouping of dipoles in this work is based on Brodmann areas - a cytoarchitectural, rather than a functional classification of the human cerebral cortex. However, even though Brodmann areas were originally defined on the basis of the cytoarchitectural organization of the neurons in the cerebral cortex, more recent studies have unveiled the structural-functional correlations of many Brodmann areas, and thus point to the potential suitability of such segmentation of the brain as a tentative basis for a functional classification relevant to $\mathrm{EE}^{35}$.

Three different EEG headset configurations with 19, 33 and 71 electrodes are used for the numerical experiments in this paper. A schematic representation of the headsets in consideration is shown in Figure 1. No human subject research was involved in the preparation of this paper, but this is planned for the next stage of our research.

\subsection{Mathematical Formulation of EEG imaging}

The equation often used for defining the forward and inverse imaging problems in EEG has the following form: 
Here $\boldsymbol{\Phi} \in \mathfrak{R}^{N_{E} \times 1}$ is a vector of the scalp electric potentials measured by the $N_{E}$ electrodes with respect to a reference electrode, $\boldsymbol{J} \in \mathfrak{R}^{3 N_{V} \times 1}$ is the primary or impressed current density vector, where $N_{V}$ is the number of dipole locations in the brain, with each dipole current vector having three independent components corresponding to the usual Cartesian coordinates in $3 \mathrm{D}$ space, $\boldsymbol{K} \in \mathfrak{R}^{N_{E} \times 3 N_{V}}$ is the lead field matrix, $c$ is a constant which embodies the fact that the electric potential is determined up to an arbitrary constant ${ }^{15}$ and $1 \in \mathfrak{R}^{\hat{N}_{E} \times 1}$ is a vector of ones.

Source localization works by first calculating the scalp potentials produced by virtual dipoles at arbitrary locations in the brain (i.e. solving the forward problem), and then, in conjunction with the actual EEG data measured by the electrodes, it is used to work back and estimate the sources that best fit the measurements (i.e. solving the corresponding inverse problem). Typically, this inverse problem is ill-posed, hence additional constraints are added in order to produce an appropriate unique solution.

\footnotetext{
r

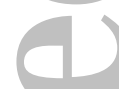

1.2 Compressed Sensing and Sparse Signal Recovery

r

Compressed sensing is an emerging field of information theory which shows that one can exploit sparsity or compressibility when acquiring signals of general interest and that one can design a non-adaptive sampling strategy that condenses the information in a compressible signal into a small amount of measurements. In a nutshell, compressed sensing proposes to find a signal $\boldsymbol{J} \in \mathfrak{R}^{N}$ by collecting $M$ linear measurements of the form $\Phi_{m}=<\boldsymbol{K}_{\boldsymbol{m}}, \boldsymbol{J}>+z_{m}, 1 \leq m \leq M$ or in matrix notation

$$
\boldsymbol{\Phi}=\boldsymbol{K} \boldsymbol{J}+\boldsymbol{z}
$$

where $\boldsymbol{K}$ is a $N \times M$ sensing matrix with $N$ usually smaller than $M$ (by one or several orders of magnitude, i.e., $N<<M$ ) and $\boldsymbol{z}$ is an error term representing measurement error. Since $N<M$, eq. (2) is an underdetermined linear system and such systems do not have a unique solution. Encouragingly, sparsity constraint is useful to find a unique solution of eq. $(2)^{36}$. In particular, one can solve the following optimization problem to find a unique solution of eq. $(2)^{37}$ :
} 
$\hat{\boldsymbol{J}}_{1}=\arg \min \|\boldsymbol{J}\|_{1} \quad$ s.t. $\quad\|\boldsymbol{\Phi}-\boldsymbol{K} \boldsymbol{J}\|_{2}<v$,

for a carefully chosen $v \neq 0$. Here $\|\boldsymbol{J}\|_{1}$ refers to the $l_{1}$-norm.

The performance of the sparse approximation algorithms depends on several properties of the projection matrix $\boldsymbol{K}$ and one important consideration for sparse approximation is the so-called coherence of the projection matrix ${ }^{38}$ which is defined as

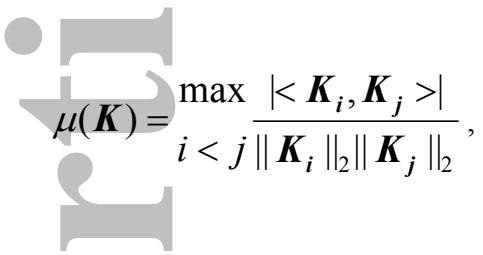

where $\boldsymbol{K}_{\boldsymbol{i}}$ and $\boldsymbol{K}_{\boldsymbol{j}}$ denote the $i$-th and $j$-th column of $\boldsymbol{K}$ respectively. We say that a matrix is incoherent if $\mu$ is

much smaller than one. Generally lower coherence ensures better reconstruction from a small number of measurements.

Sparse signal recovery algorithms can be categorized into greedy algorithms $\mathrm{s}^{39}$, algorithms based on mixed norm optimization $^{40}$, iterative reweighted algorithms ${ }^{41}$ and Bayesian algorithms ${ }^{42}$. Sparse Bayesian Learning (SBL) methodology ${ }^{41}$ has been found very promising for solving the inverse problems when the projection matrix is highly coherent ${ }^{29}$, which is typical for EEG. Considering the highly coherent nature of the lead-field matrix in this work we have used $\mathrm{SBL}^{41}$ to solve eq. (3) in conjunction with the Brodmann map based segmentation of the cerebral cortex. A number of algorithms based on SBL also consider incorporating temporal correlation of the sources $^{43}$ to ensure better performance, however in this work we do not consider any temporal correlations because of the additional complexity of computing and then efficiently incorporating such information, which could be a computationally demanding task ${ }^{43}$.

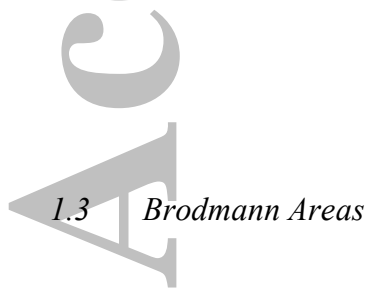

Brodmann areas were originally defined by the German anatomist Korbinian Brodmann in 1909 based on the cytoarchitectural organization of neurons that he observed in the cerebral cortex as a result of a careful microscopic examination ${ }^{44}$. Brodmann's map of the human cortex contains 43 cytoarchitectonic areas, which are labeled by numbers between 1 and 52. Areas with the numbers 12-16 and 48-51 are not used in his map of the 
human brain $^{35}$. Brodmann explained these 'gaps' by the fact that some areas are not identifiable in the human cortex but are well developed in other mammalian species. Brodmann areas have been discussed, debated, refined, and renamed exhaustively for more than a century. In the 1980s Brodmann's map gained a renewed popularity with the introduction of novel functional and structural neuroimaging techniques, which allowed the translation of the two-dimensional information of the original map into a three-dimensional representation ${ }^{35}$. The goal of the Brodmann's map was to produce a comparative organic theory of the cerebral cortex based on anatomical features ${ }^{35}$. This hypothesis could not be rigorously tested in his time, except for some so-called primary areas such as the primary visual cortex. Recent functional imaging studies have demonstrated that in many cases this anatomical segmentation of the human brain is valid for its functionality as well, although there are studies demonstrating that the map could be incomplete in other cases ${ }^{35}$.

It is already well accepted that neurons in the brain work in groups or clusters, however no precise map is currently available that would be able to group the neurons accurately in regard to brain functionality. We therefore rely in the present work on the most widely known and accepted cytoarchitectural segmentation of the human cortex represented by the Brodmann's map.

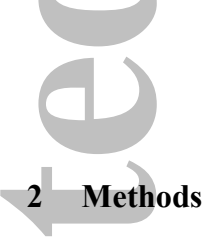

In order to better localize the sources of electrical activity inside human brain the proposed model relies on the following two steps

- Firstly it considers grouping the dipoles into several zones based on functionality. Such consideration reduces the search space significantly and thus points to more accurate activity localization.

- Secondly it applies TMSBL ${ }^{43}$ algorithm to reconstruct the sparse signal. It relies on the hypothesis that the signal is sparse when it is presented in terms of the active Functional Zones.

\subsection{Data Model and Assumptions}

A realistic head model obtained from the segmentation of MRI images of the head was used for the experiment. suitable for solution of the direct and inverse EEG problems The model included four different major components, namely scalp, skull, CSF and brain, with the following relative conductivity values ${ }^{9}: \sigma_{\text {scalp }}=1$, 
$\sigma_{\text {skull }}=0.05, \sigma_{\mathrm{CSF}}=5, \sigma_{\text {brain }}=1$. The source space was constructed by dividing the head model into cubes with a size of $5 \times 5 \times 5 \mathrm{~mm}^{3}$ and considering possible current dipoles only in the center of those cubes that consisted of at least $60 \%$ of gray matter. The head model was defined on a discrete grid with a grid step of $1.25 \mathrm{~mm}$. This segmentation procedure resulted in 6203 dipole positions ${ }^{18}$. As of volume conduction model we used Finite Difference Method (FDM) ${ }^{45}$. Worth mentioning, with FDM volume conduction model, the mapping between MRI images and FDM pixels are very straight forward ${ }^{45}$.

In order to implement the proposed sparsity criteria, the considered 6203 dipoles were clustered according to Brodmann areas. While the number of Brodmann areas for the human cortex is $43^{35}$, the above mentioned segmentation procedure produced no dipoles in BA 26 and thus resulted in 42 Brodmann areas in total. Considering both the left and the right hemisphere, we ended up with 84 dipole clusters which we called Functional Zones. We assumed that all the dipoles (or most of the dipoles) in a particular Functional Zones are activated simultaneously during specific tasks performed by the human brain. The considered 84 Functional Zones were used to recompute the previously calculated lead field matrix from $\boldsymbol{K} \in \mathfrak{R}^{N_{E} \times(3 \times 6203)}$ to $\boldsymbol{K}_{\boldsymbol{F Z}} \in \mathfrak{R}^{N_{E} \times(3 \times 84)}$, by taking the average of all the lead field values belonging to each group in regard to $x, y$ and $z$ components of each electric current dipole.

In general, dipoles can be of different strength and randomly oriented, however in the present experiment, we considered for simplicity that the active dipoles had unit length and were vertically oriented. Electrode locations corresponding to a 19-channel Compumedics (Setup I) and 14-channel Emotiv EPOC (Setup II) headsets were used for the study.

\subsection{Localizing the Sources of Electrical Activity}

Sparse Bayesian Learning (SBL) methodology ${ }^{46}$ is used to find the inverse solution. SBL was initially proposed for regression and classification by Tipping ${ }^{42}$ in machine learning. In ${ }^{46}$, Wipf et al. applied SBL for the sparse signal recovery problem. The idea of SBL is to find $\boldsymbol{J}$ through Maximum a Posterior (MAP) estimate ${ }^{43}$. Several variants of SBL algorithm are found in the literature ${ }^{47}$, and in this work we consider the $\mathrm{TMSBL}^{43}$ algorithm, which is found superior when the dictionary matrix is highly coherent ${ }^{29}$. While TMSBL is derived for Multiple Measurement Vector (MMV) model, it can be used in the Single Measurement Vector (SMV) model (i.e. our 
case). In this case $\mathrm{TMSBL}^{43}$ is similar to $\mathrm{EM}_{-\mathrm{SBL}^{46}}$ with the key difference in the error variance $\left(\sigma^{2}\right)$ learning rule $^{47}$. The parametric form of the SBL weight prior here is given by

$$
p(\boldsymbol{J}, \boldsymbol{\gamma})=\prod_{i=1}^{M}\left(2 \pi \gamma_{i}\right)^{-\frac{1}{2}} \exp \left(-\frac{J_{i}^{2}}{2 \gamma_{i}}\right)
$$

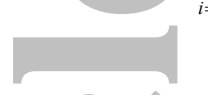

where $\boldsymbol{\gamma}=\left[\gamma_{1}, \ldots, \gamma_{\mathrm{M}}\right]^{T}$ is a vector of $M$ hyperparameters controlling the prior variance of $\boldsymbol{J}$. These

hyperparameters along with the error variance $\left(\sigma^{2}\right)$ are estimated from the data by marginalizing over $\boldsymbol{J}$ and then performing maximum likelihood optimization ${ }^{46}$. The marginalized probability density function is given by

$$
p\left(\boldsymbol{\Phi}, \boldsymbol{\gamma}, \sigma^{2}\right)=\int p\left(\boldsymbol{\Phi} \mid \boldsymbol{J} ; \sigma^{2}\right) p(\boldsymbol{J} ; \boldsymbol{\gamma}) d \boldsymbol{J}
$$
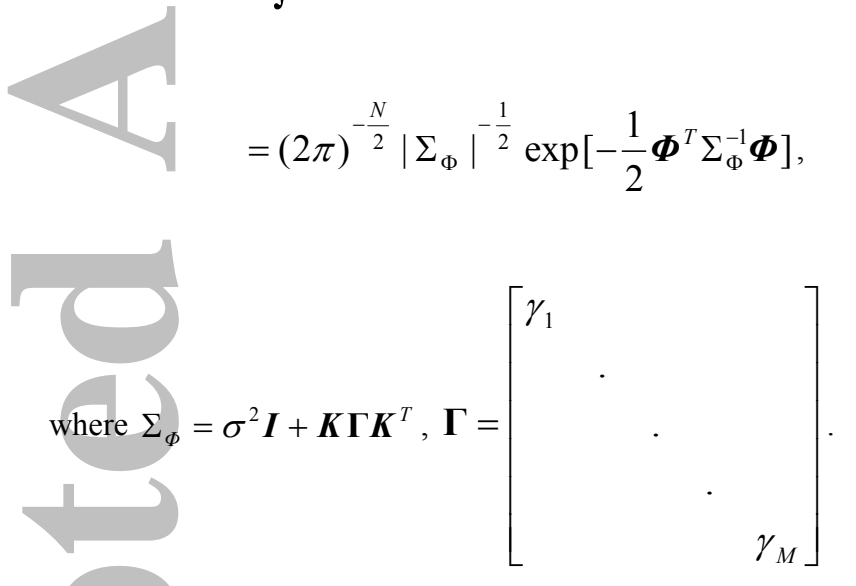

We now need to estimate $\gamma$ and $\sigma^{2}$ from the data and for this we employ EM algorithm ${ }^{43}$. Once we have these values the solution of the inverse problem is computed as

$$
\hat{\boldsymbol{J}}=\left(\boldsymbol{K}^{T} \boldsymbol{K}+\sigma_{\mathrm{ML}}^{2} \boldsymbol{\Gamma}_{\mathrm{ML}}^{-1}\right)^{-1} \boldsymbol{K}^{T} \boldsymbol{\Phi}
$$

The solution $\hat{\boldsymbol{J}} \in \mathfrak{R}^{(3 \times 84) \times 1}$ is $a(3 \times 84) \times 1$-is a component vector represents current sources at the 84 locations within the brain volume with three directional (i.e. $x, y, z$ directions) components per location. Each location

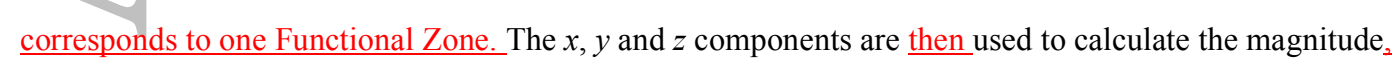

$d=\sqrt{x^{2}+y^{2}+z^{2}}$ of the current density for each of the Functional Zones. We thus form a vector $\underline{V \in \mathfrak{R}^{84 \times 1}} \underline{\text { representing the From the magnitudes of the current density of the Functional Zones. From } V}$, the 
maximum magnitude, $\underline{\max }_{\text {max_Mag }} M$ is determined. Any Functional Zone with a magnitude larger than or equal to $t h r \times \underline{V}_{\text {max }}$ Of Max_Mag, is considered to be active ${ }_{2}=$ Here The threshold, $t h r$ is a threshold which is

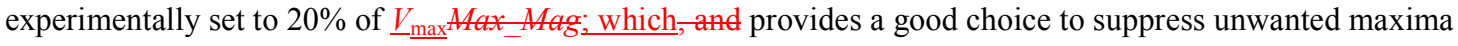
(if there is any) while keeping the actual onesmaxima.

\section{Experiments and Results}

1.

Experiments were conducted by varying the number of simultaneously active Functional Areas for three EEG headset configurations as specified in the 'Introduction' section. The idea of using different electrodes setups for the experiment was to analyse how the reconstruction performance of the proposed approach varies from low density to high density headsets.

Error Distance (ED) ${ }^{48}$ was used to analyse the reconstruction performance. The error distance between the actual and the estimated source locations is defined as

$$
E D=\frac{1}{N_{I}} \times \sum_{i \in I}^{N_{I}} \min _{l}\left\|\boldsymbol{r}_{i}-\boldsymbol{s}_{\boldsymbol{l}}\right\|+\frac{1}{N_{L}} \times \sum_{l \in L}^{N_{L}} \min _{i}\left\|\boldsymbol{r}_{l}-\boldsymbol{s}_{\boldsymbol{i}}\right\| .
$$

Here $\boldsymbol{s}_{\boldsymbol{l}}$ and $\boldsymbol{r}_{\boldsymbol{i}}$ are the actual and estimated source locations respectively. $N_{I}$ and $N_{L}$ are the total numbers of estimated and the undetected sources respectively. The first term of equation (5) calculates the mean of the distance from each estimated source to its closest real source, and the corresponding real source is then marked as detected. All the undetected real sources made up the elements of the data set $L$ and thus the second term of the equation calculates the mean of the distance from each of the undetected sources to the closest estimated

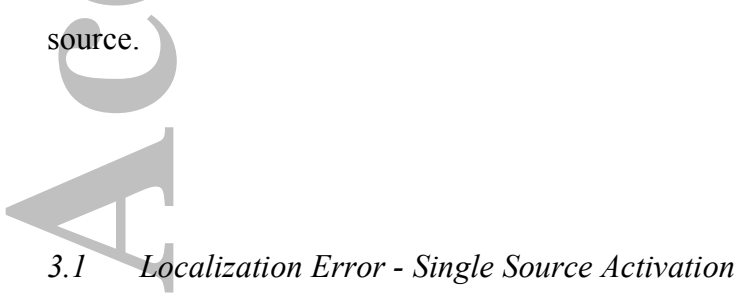

First, a single Functional Zone was considered active and the corresponding potentials on the electrodes were calculated based on $\boldsymbol{K}_{\boldsymbol{F Z}}$. The experiment was conducted for all Functional Zones activated sequentially one at a time. Localization of the Functional Zone was performed according to the algorithm specified above in section 
'Localizing the Sources of Electrical Activity'. With due interest, we also performed the source reconstruction using sLORETA for the lead field matrix $\boldsymbol{K}_{\boldsymbol{F Z}}$. For this experiment, each Functional Zone was represented by its centroid $\boldsymbol{s}_{\boldsymbol{i}}\left(\forall_{i} \in 1: \boldsymbol{F Z}\right)$. For an active source position $\boldsymbol{s}_{\boldsymbol{i}}$ we claimed a "success" if the error distance was

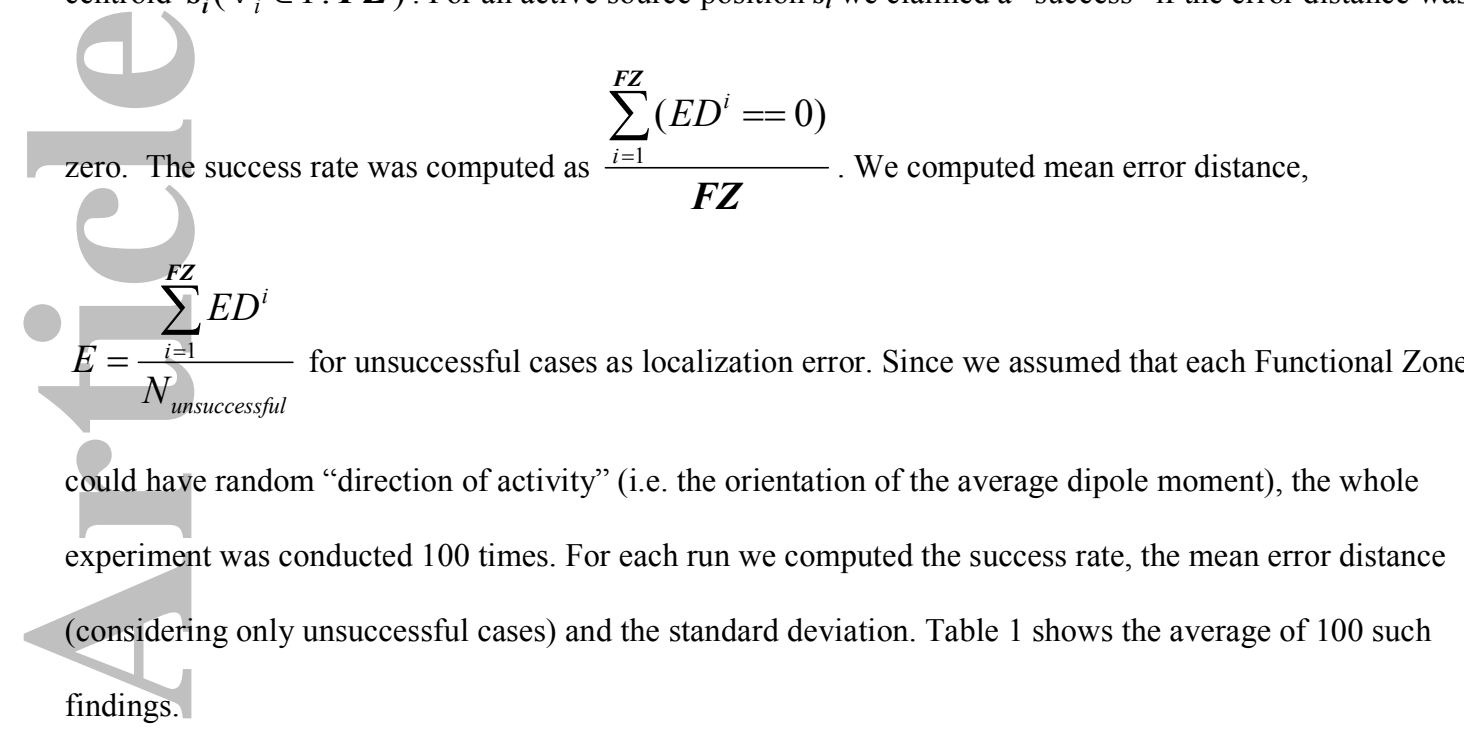

From the results it is observable that for a single active Functional Zone the percentage of exact localization by the proposed method is quite high, and for the 71 electrodes setup it is $100 \%$. For sLORETA, the localization performance is quite poor, and to our understanding it is mainly due to the broadly distributed sources produced by sLORETA, which resulted in multiple active Functional Zones to be detected in the reconstructed signal. It is worth mentioning that experimentally, we figured out that considering only one Functional Zone with the maximum magnitude, sLORETA produces a success rate of $100 \%$, which is consistent with the finding that sLORETA produces zero localization error for a single active source ${ }^{17}$.

3.2 Localization Error - Multiple Sources Activation

In this case $S(S>1)$ Functional Zones were activated simultaneously. From the total of $C_{s}^{84}=\frac{84 !}{S !(84-S) !}=\frac{84(84-1)(84-2) \ldots(84-S+1)}{S !}$ possible combinations of 84 Functional Zones, one combination was chosen randomly. For each value of $S$ we claimed a "success" if the error distance was zero. Since Functional Zones could have random directions of activity, the experiment was performed 1000 times and the success rate was computed over these 1000 runs for each value of $S$. For the unsuccessful cases we 
computed the mean error distance, $E=\frac{\sum_{i=1}^{1000} E D^{i}}{N_{\text {unsuccessful }}}$ and the standard deviation of the mean error distance for each value of $S$. For comparison, we performed the same experiment where we applied sLORETA instead of TMSBL to reconstruct the sparse signal. Figure 5 shows the success rates as a function of $S$. In case of

sLORETA based reconstruction even for 2 simultaneously active Functional Zones and for 71 electrodes setup, the success rate of exact localization was zero. Hence the success rate of sLORETA is omitted in Figure 5.

Figure 6 shows the localization errors for unsuccessful cases with bars showing the mean error distance and whiskers showing the standard deviation.

From the results it is observable that the success rate decreases with the increased number of simultaneously active zones. Unsurprisingly, better localization accuracy was found for the 71-electrodes setup in comparison to other setups. For the proposed method, even for 6 simultaneously active Functional Zones, the 71-electrodes setup was able to locate all the active zones accurately in around $70 \%$ of the cases, and for the rest $30 \%$ of the cases, it produced localization errors of about $15 \mathrm{~mm}$. For 33-electrodes setup and up to 3 simultaneously active Functional Zones, the proposed approach was found to locate the active zones accurately for greater than $70 \%$ of the cases (with a localization error of $<=15 \mathrm{~mm}$ for unsuccessful cases). Evidently, larger numbers of simultaneously active Functional Zones result in less accurate localizations. For 19-electrodes setup, the proposed approach produced accurate localization only for $40 \%$ of the cases even only for 2 simultaneously active Functional Zones with a localization error of about $21 \mathrm{~mm}$ (for the rest $60 \%$ of the cases).

3.3 Partially activated Functional Zone

This part of the experiment was designed to analyse the performance of the proposed method when only a fraction of the Functional Zone rather than the whole Functional Zone was active. For each of the activated Functionall Zone a specified percentage of the dipoles belonging to that Functional Zone were activated. One dipole of the considered Functional Zone was chosen randomly and the rest of the active dipoles (based on the specified percentage of activated dipoles) were adjacent to the chosen dipole. The Lead field matrix $\boldsymbol{K}$ was used for the forward problem (i.e. to generate $\boldsymbol{\Phi}$ ), whereas $\boldsymbol{K}_{F Z}$ was used for the inverse problem. While Functional Zone(s) can have random direction of activity, all the considered dipoles within a given Functional Zone were 
considered to have the same orientation. The experiment was conducted for all the Functional Zones (1: FZ) activated sequentially, one at a time, for the 33 electrode setup. In this case we claimed a "success" if the activated Functional Zone was exactly detected in the reconstructed signal (i.e. if the error distance was zero).

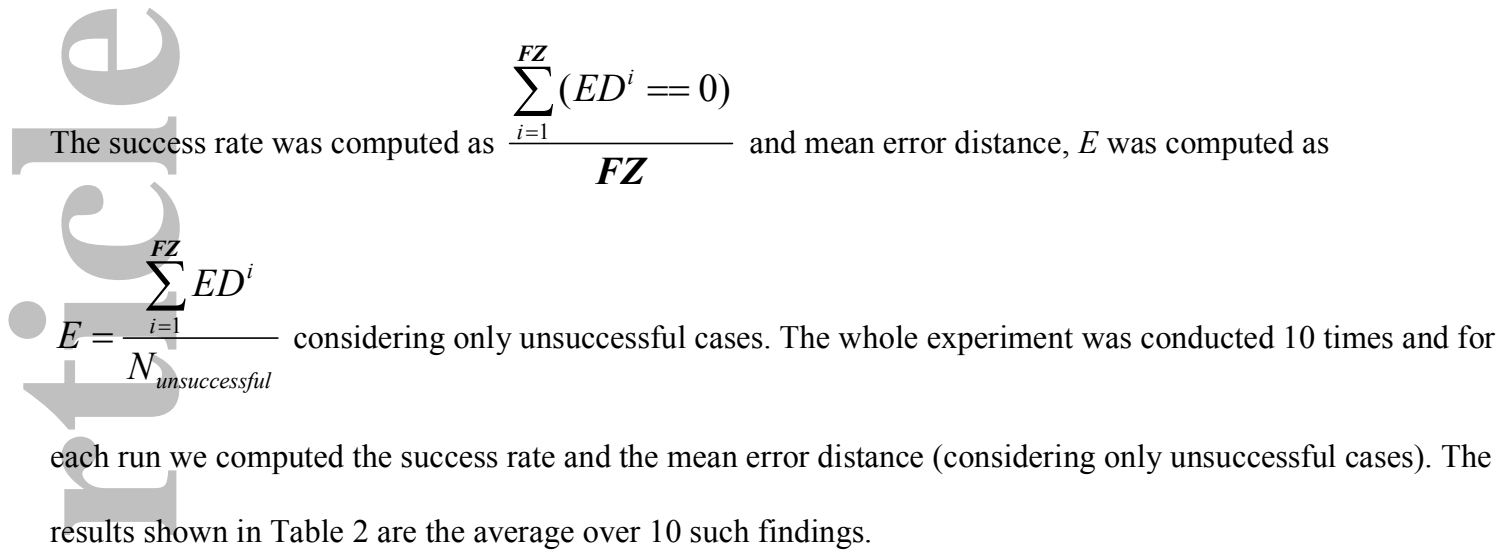

From the results it is clear that for the proposed method when the whole area of the considered zone is active, it is very likely that it will be exactly localized in the reconstructed signal. As soon as the percentage of the active area decreases the chances of inexact localization increases. Interestingly for sLORETA, the performance remains steady with respect to the percentage of activation. It has already been explored that sLORETA produces zero localization error for a single active dipole, however produces broad distribution; thus $50 \%$ activation or $100 \%$ activation of the Functional Zone does not matter much. Another important observation is that for the proposed method, when the percentage of activation decreases, one could have more than one active Functional Zone in the reconstructed signal, however it is very likely that the activity maxima will still point to the actual activated Functional Zone. In order to verify that claim, rather than computing the error distance as in Table 2, we considered only one Functional Zone having the maximum magnitude in the reconstructed signal, and then computed the Euclidian distance between the actual and the estimated Functional Zone. The findings are shown in Table 3.

are shos

For the proposed method, significant increase of the success rate is observable. For sLORETA, one might expect $100 \%$ success rate even for $50 \%$ activation of the Functional Zone, however a slight decrease of the success rate is observable. To our understanding, this is due to the fact that for a smaller Functional Zone having smaller percentage of activation, the number of active dipoles can be even down to 1 and when such dipole is 
located at the border of Functional Zone, there is a high possibility that an adjacent Functional Zone could be detected as active instead of the actual active Functional Zone.

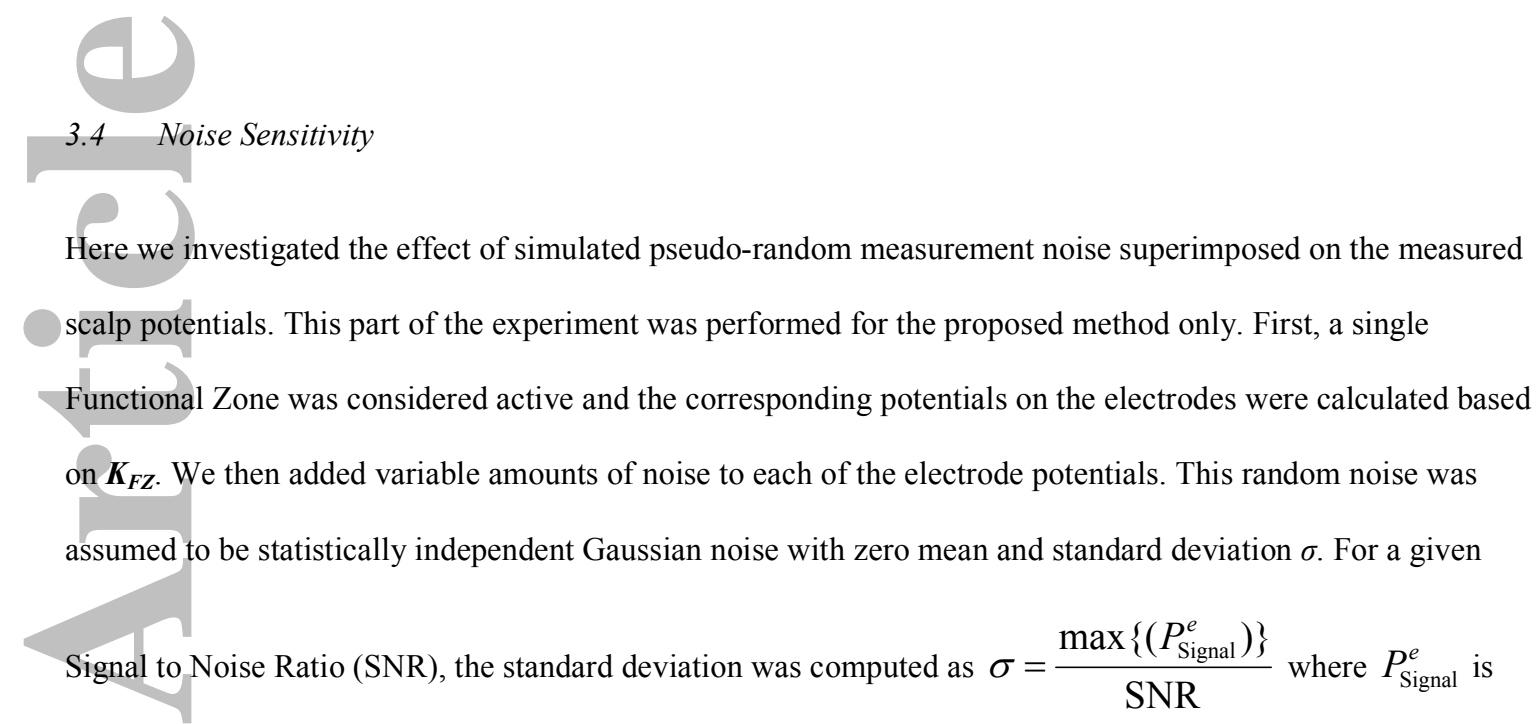

the signal measured by the electrode $e\left(\forall_{e} \in 1: N_{E}\right)$. The experiment was conducted for all Functional Zones ractivated sequ activated sequentially one at a time and for different levels of $\operatorname{SNR}(5,10,15,20,25,30$ and infinity).

Localization of the Functional Zone was performed according to the algorithm specified above in section 'Localizing the Sources of Electrical Activity'. As we were adding a random amount of noise, the above mentioned ex

mentioned experiment was conducted 100 times for each Functional Zone, $\boldsymbol{s}_{\boldsymbol{i}}\left(\forall_{i} \in 1: \boldsymbol{F Z}\right)$. Figure 7 shows the success rate of the experiment for different electrodes setups and different levels of SNR.

From the results it is observable that the success rate decreases with the increase in the amount of noise.

Unsurprisingly, the 71-electrodes setup is the most robust against noise in localizing the sources of activity accurately. For the 71-electrodes setup the proposed approach was found $>50 \%$ successful even with SNR of 5 . For 31 - and 19- electrodes setup the proposed method produced success rate of greater than and around $50 \%$ for the SNR of 15 .

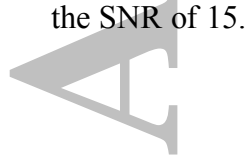

\subsection{Analysis of the lead-field matrices (Conditioning of the reconstruction)}

Note that equation (1) is ill conditioned in two respects. Firstly, it is under determined in the sense that there are fewer equations than unknowns, although, to a certain extent, this is ameliorated here by the assumption that the 
solution is sparse. Secondly the columns of the lead field matrix, $\boldsymbol{K}$ are highly coherent, which suggests that the error in the data will be amplified when solving for $\boldsymbol{J}$.

For $l_{2}$ approximation, such ill conditioning can be analysed via the singular value decomposition of the lead field matrix $\boldsymbol{K}^{49}$, namely $\boldsymbol{K}=\boldsymbol{U} \boldsymbol{S} \boldsymbol{V}^{T}$ where $\boldsymbol{S}=\operatorname{diag}\left(s_{1}, s_{2}, \ldots, s_{N_{E}}\right), s_{1} \geq \ldots \geq s_{N_{E}} \geq 0$, and $\boldsymbol{U} \in \mathfrak{R}^{N_{E} \times N_{E}}$,

$\boldsymbol{V} \in \mathfrak{R}^{N_{E} \times 3 N_{V}}$ satisfy $\boldsymbol{U} \boldsymbol{U}^{T}=\boldsymbol{I}$ and $\boldsymbol{V} \boldsymbol{V}^{T}=\boldsymbol{I}$. The singular values for the lead field matrix based on the Brodmann areas are given in Figure 8. Although we are interested in $l_{1}$ approximation rather than $l_{2}$ approximation, it is plausible that the singular values should play a role here also.

(1)

To investigate this, we note that equation (1) can be rewritten as

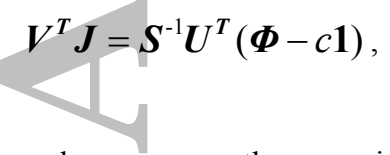

and we compare the errors in (1) to the effect of errors in (6). Specifically, we compare the effect of the perturbed equation

$\boldsymbol{K} \boldsymbol{J}=\boldsymbol{\Phi}+\alpha^{-1}\|\boldsymbol{\Phi}\|_{2} \boldsymbol{\varepsilon}$,

or, equivalently,

$\boldsymbol{V}^{\boldsymbol{T}} \boldsymbol{J}=\boldsymbol{S}^{-1} \boldsymbol{U}^{\boldsymbol{T}} \boldsymbol{\Phi}+\beta^{-1}\left\|\boldsymbol{S}^{-1} \boldsymbol{U}^{\boldsymbol{T}} \boldsymbol{\Phi}\right\|_{2} \boldsymbol{\varepsilon}$

Here $\boldsymbol{\Phi}$ is the measured potential when only one of the Brodmann areas is active, the components of $\boldsymbol{\varepsilon}$ are mean zero independently and identically distributed random numbers with variance $\frac{1}{N_{E}}, \alpha$ is the signal to noise ratio for equation (7) and $\beta$ is the signal to noise ratio for equation (8).

We have performed a number of simulations aimed at quantifying the accuracy of identifying which Brodmann area is active when only one area is active and we have found that the accuracy for (7) with the signal to noise ratio $\alpha=10$ when the number of electrodes is 33 gives comparable results to (8) when $\beta=2.2$. A simple analysis indicates that, for the general case, the performance of (7) and (8) are the same when $\beta=\kappa \alpha$, where 


$$
\kappa=N_{E}\left(\sum_{i=1}^{N_{E}} s_{i}{ }^{2}\right)^{\frac{1}{2}}\left(\sum_{i=1}^{N_{E}} s_{i}{ }^{-2}\right)^{\frac{1}{2}}
$$

Thus, the term $\kappa$ provides a measure of the effect of conditioning of the lead field matrix.

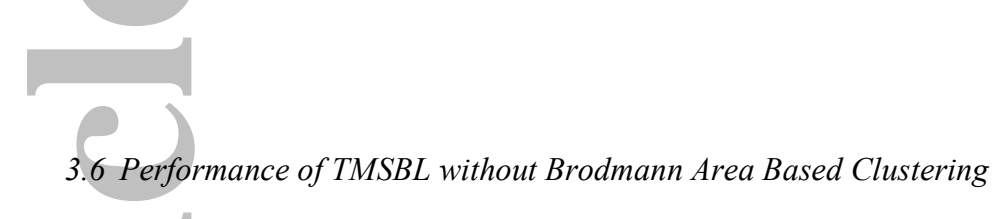

In this experiment, we evaluated the performance of the TMSBL method without considering the grouping of the dipoles. The lead filed matrix $\boldsymbol{K}$ was used to generate the forward problem, and to solve the inverse problem. First, a single dipole was considered active and the corresponding potentials on the electrodes were calculated based on $\boldsymbol{K}$. The experiment was conducted for all the dipoles activated sequentially, one at a time. For an active dipole position $s_{i}$ a "success" was claimed if the error distance was zero. The success rate was computed as $\frac{\sum_{i=1}^{6203}\left(E D^{i}==0\right)}{6203}$ The mean error distance, $E=\frac{\sum_{i=1}^{6203} E D^{i}}{N_{\text {unsuccessful }}}$ was computed for unsuccessful cases as localization error. Since we assumed that each dipole could have a random "direction of activity" (i.e. the orientation of the dipole moment), the whole experiment was conducted 100 times. For each run, the success rate, the me rate, the mean error distance (considering only unsuccessful cases) and the standard deviation were computed. Table 4 shows the average of 100 such findings.

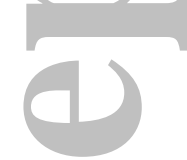

\section{Discussion}

Recent years have witnessed a tremendous interest to sparse approximation in signal processing. Sparsity constraints have also been considered in EEG for localizing the sources of electrical activity inside the brain, i.e. for solving the inverse EEG problem. Considering that the number of dipoles in a realistic head model is usually significantly higher than the typical number of electrodes in an EEG headset, and the electrical signals spread very significantly on propagation through the head, this inverse problem is highly ill-posed, and the best algorithms are able to solve the problem only for a very limited number of simultaneously active dipoles. It is already well accepted that usually a group of adjacent dipoles rather than a single dipole gets activated simultaneously. Hence in order to better specify the sparsity profile, our proposed method groups the considered 
dipoles in the human cerebral cortex into several Functional Zones, each zone corresponding to one of the Brodmann's areas, and then applies the sparsity constraint to these Functional Zones rather than individual dipoles. It is worth mentioning the number of runs for each individual experiment has been determined
experimentally and has been set to a number so that the mean score obtained does not change very much even
with larger number of runs.

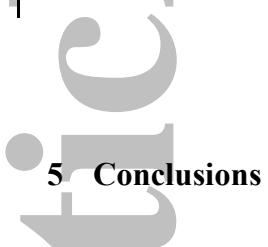

In this paper, we have proposed an innovative source localization method for EEG where the method was tested by varying the number of simultaneously activated Functional Zones and with different electrode setups. The results indicate that the proposed method is quite promising in locating the activated Functional Zones accurately. Even after downsizing the lead field matrix, sLORETA was found to be quite inadequate to localize multiple active sources. For a 71-electrodes setup and up to 2 simultaneously activated Functional Zones, the proposed model can locate the sources of activation exactly in the noiseless case. Even though the percentage of exact localization decreases with the increase of the number of simultaneously active zones, the percentage of exact localization is still around $70 \%$ for up to 6 simultaneously activated Functional Zones. Unsurprisingly, for the 31- and the 19-electrodes setups, the localization accuracy is not as high as for the 71-electrodes setup; however when the signal is highly sparse (e.g. up to 4 simultaneously activated Functional Zones for 31electrodes setup, and up to 2 simultaneously activated Functional Zones for 19-electrodes setup), these setups are found to produce fairly accurate localization. Since the relevant details of the human brain activity are still not known precisely, it is particularly important to know what percentage of the area of a Functional Zone needs to be activated for successful localization. Experiments reveal that the proposed model is fairly accurate to locate the sources of activity even with $50 \%$ activation of the Functional Zone. The experiment also reveals a significant improvement of the TMSBL method for EEG source localization when the lead field matrix was down sized by the clustering of the dipoles.

While the proposed model is found quite promising in accurately localizing the activated Brodmann areas in a simulation environment, it is yet to be found how the proposed approach will perform with real data (i.e. localizing the brain activity of a subject). Our future work will also aim at analysing the performance of the proposed approach with respect to different brain atlases, for example the $\mathrm{AAL}^{50}$ and on evaluating the 
correlation of the EEG-based activity localization with that of fMRI.

\section{Acknowledgment}

This project was supported by the Computational and Simulation Sciences Transformational Capability Platform of Commonwealth Scientific and Industrial Research Organisation (CSIRO), Australia, along with University of New South Wales (UNSW), Canberra, Australia. The authors would like to thank Dr. Chao Suo and Dr. Roger Koenig-Robert of Monash Biomedical Imaging (MBI) Laboratory, VIC, Australia for their valuable comments and suggestions.

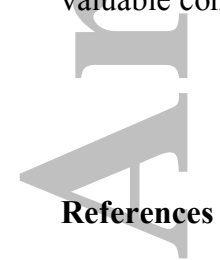

[1] Jervis BW, Coelho M, Morgan GW. Spectral analysis of EEG responses. Medical and Biological Engineering and Computing May. 1989; 27 (3): 230-238.

[2] Plummer C, Harvey AS, Cook M. EEG source localization in focal epilepsy: Where are we now? Epilepsia. 2008; 49 (2): 201-218.

[3] Xie S, Krishnan S. Wavelet-based sparse functional linear model with applications to EEGs seizure detection and epilepsy diagnosis. Medical and Biological Engineering and Computing. 2013; 51 (1-2): 49-60.

[4] Crevecoeur G, Hallez H, Van Hese P, D’Asseler Y, Dupré L, Walle R. A hybrid algorithm for solving the EEG inverse problem from spatio-temporal EEG data. Medical and Biological Engineering and Computing. 2008; 46 (8): 767-777.

[5] Finnigan S, Putten MJ. EEG in ischaemic stroke: quantitative EEG can uniquely inform (sub-)acute prognoses and clinical management. Clin Neurophysiol. 2013: 124 (1); 10-19.

[6] Phan TG, Gureyev T, Nesterets Y, Ma H, Thyagarajan D. Novel Application of EEG Source Localization in the Assessment of the Penumbra. Cerebrovascular diseases. 2012; 33 (4): 405-407.

[7] Kaplan PW, Rossetti AO. EEG Patterns and Imaging Correlations in Encephalopathy: Encephalopathy Part II. Journal of Clinical Neurophysiology. 2011; 28 (3): 233-251.

[8] Grech R, Cassar T, Muscat J, et al. Review on solving the inverse problem in EEG source analysis. Journal of neuroengineering and rehabilitation. 2008; 5 (1). 
[9] Nunez PL, Srinivasan R. Electric fields of the brain: the neurophysics of EEG. Oxford University Press, USA, 2006.

[10] Pascual-Marqui RD. Review of methods for solving the EEG inverse problem. International journal of bioelectromagnetism. 1999; 1 (1): 75-86.

[11] Gençer NG, Williamson SJ. Differential characterization of neural sources with the bimodal truncated SVD pseudo-inverse for EEG and MEG measurements. IEEE Transactions on Biomedical Engineering. 1998; 45 (7): $827-838$.

[12] Pinto B, Silva CQ. A simple method for calculating the depth of EEG sources using minimum norm estimates (MNE). Medical and Biological Engineering and Computing. 2007; 45 (7): 643-652.

[13] Silva C, Maltez JC, Trindade E, Arriaga A, Ducla-Soares E. Evaluation of L1 and L2 minimum norm performances on EEG localizations. Clinical neurophysiology. 2004; 115 (7): 1657-1668.

[14] Cheng LK, Bodley JM, Pullan AJ. Comparison of potential-and activation-based formulations for the inverse problem of electrocardiology. IEEE Transactions on Biomedical Engineering. 2003; 50 (1): 11-22.

[15] Pascual-Marqui RD. Standardized low-resolution brain electromagnetic tomography (sLORETA): technical details. Methods Find Exp Clin Pharmacol. 2002; 24 (Suppl D): 5-12.

[16] Gramfort A, Kowalski M, Hämäläinen M. Mixed-norm estimates for the M/EEG inverse problem using accelerated gradient methods. Physics in medicine and biology. 2012; 57 (7).

[17] Wagner M, Fuchs M, Kastner J. Evaluation of sLORETA in the presence of noise and multiple sources. Brain Topography. 2004; 16 (4): 277-280.

[18] Saha S, Nesterets YI, Tahtali M, Gureyev TE. Evaluation of spatial resolution and noise sensitivity of sLORETA method for EEG source localization using realistic head model. Biomedical Physics \& Engineering Express. 2015; 1 (4): 045206.

[19] Gorodnitsky IF, George JS, Rao BD. Neuromagnetic source imaging with FOCUSS: a recursive weighted minimum norm algorithm. Electroencephalography and clinical Neurophysiology. 1995; 95 (4): 231-251.

[20] Li Y. A globally convergent method for lp problems. SIAM Journal on Optimization. 1993; 3(3): 609-629.

[21] Daubechies I, DeVore R, Fornasier M, Gunturk S. Iteratively re-weighted least squares minimization: proof of faster than linear rate for sparse recovery. 42nd Ann. Conf. on Inf. Sci. Syst. 2008; 26-29.

[22] Osborne MR, Presnell B, Turlach BA. A new approach to variable selection in least squares problems. IMA journal of numerical analysis. 2000; 20(3): 389-403. 
[23] Tibshirani R. Regression shrinkage and selection via the lasso. Journal of the Royal Statistical Society Series B (Methodological). 1996; 267-288.

[24] Efron B, Hastie T, Johnstone I, Tibshirani R. Least angle regression. The Annals of statistics. 2004 ; 32 (2): 407-499.

[25] Friedman J, Hastie T, Hofling H, Tibshirani R. Pathwise coordinate optimization. Ann. Appl. Stat. 2007; 1: $302-32$

[26] Bruce AG, Sardy S, Tseng P. Block coordinate relaxation methods for nonparamatric signal denoising, Aerospace/Defense Sensing and Controls. International Society for Optics and Photonics. 1998.

[27] Donoho DL. Compressed sensing. IEEE Transactions on Information Theory. 2006, 52 (4): 1289-1306.

[28] Candes E, Tao T. The Dantzig selector: statistical estimation when $\mathrm{p}$ is much larger than $\mathrm{n}$. The Annals of Statistics. 2007; 2313-2351.

[29] Ding L, Yuan H. Simultaneous EEG and MEG source reconstruction in sparse electromagnetic source imaging. Human brain mapping. 2013; 34 (4): 775-795.

[30] Ding L. Reconstructing cortical current density by exploring sparseness in the transform domain. Physics in medicine and biology. 2009; 54 (9): 2683.

[31] Wei-Tang C, Nummenmaa A, Jen-Chuen H, Fa-Hsuan L. Spatially sparse source cluster modeling by compressive neuromagnetic tomography. Neuroimage. 2010; 53 (1): 146-160.

[32] Zhang Z. Comparison of Sparse Signal Recovery Algorithms with Highly Coherent Dictionary Matrices: The Advantage of T-MSBL. Research Note. 2012.

[33] Wu SC, Swindlehurst AL. Matching Pursuit and Source Deflation for Sparse EEG/MEG Dipole Moment Estimation. IEEE Transactions on Biomedical Engineering. 2013; 60 (8): 2280 - 2288.

[34] Friston K, Harrison L, Daunizeau J, Kiebel S, et al. Multiple sparse priors for the M/EEG inverse problem NeuroImage. 2008; 39 (3): 1104-1120.

[35] Zilles K, Amunts K. Centenary of Brodmann's map — conception and fate. Nature Reviews Neuroscience. 2010; 11 (2): 139-145.

[36] Donoho DL, Elad M. Optimally sparse representation in general (nonorthogonal) dictionaries via $\ell 1$ minimization. Proceedings of the National Academy of Sciences. 2003; 100 (5): 2197-2202.

[37] Chen SS, Donoho DL, Saunders MA. Atomic decomposition by basis pursuit. SIAM J. Sci. Comput. 1998; 20 (1): 33-61. 
[38] Candes EJ, Eldar YC, Needell D, Randall P. Compressed sensing with coherent and redundant dictionaries. Applied and Computational Harmonic Analysis. 2011; 31 (1): 59-73.

[39] Tropp JA, Gilbert AC, Strauss MJ. Algorithms for simultaneous sparse approximation Part I: Greedy pursuit. Signal Processing. 2006; 86 (3): 572-588.

[40] Negahban SN, Wainwright MJ. Simultaneous Support Recovery in High Dimensions: Benefits and Perils of Block $\ell 1 / \ell \infty$-regularization. IEEE Transactions on Information Theory. 2011; 57 (6): 3841-3863.

[41] Wipf D, Nagarajan S. Iterative reweighted $\ell 1$ and $\ell 2$ methods for finding sparse solutions. IEEE Journal of Selected Topics in Signal Processing. 2010; 4(2): 317-329.

[42] Tipping ME. Sparse Bayesian learning and the relevance vector machine. The Journal of Machine Learning Research. 2001; 1: 211-244.

[43] Zhang Z, Rao BD. Sparse signal recovery with temporally correlated source vectors using sparse Bayesian learning. IEEE Journal of Selected Topics in Signal Processing. 2011; 5(5): 912-926.

[44] Brodmann K. Vergleichende Lokalisationslehre der Großhirnrinde in ihren Prinzipien dargestellt auf Grund des Zellenbaues (Barth, Leipzig, 1909); English translation available in Garey, L. J. Brodmann's Localization in the Cerebral Cortex (Smith Gordon, London, 1994), 1909.

[45] Jatoi MA, Kamel N, Malik AS, Faye I, Bornot JM, Begum T. EEG-based brain source localization using visual stimuli. International Journal of Imaging Systems and Technology. 2016; 26 (1): 55-64.

[46] Wipf DP, Rao BD. Sparse Bayesian learning for basis selection. IEEE Transactions on Signal Processing. 2004; 52 (8): 2153-2164.

[47] Zhang Z, Rao BD. Clarify some issues on the sparse Bayesian learning for sparse signal recovery. University of California, San Diego, Tech. Rep, 2011.

[48] Yao J, Dewald J. Evaluation of different cortical source localization methods using simulated and experimental EEG data. Neuroimage. 2005; 25 (2): 369-382.

[49] Golub GH, Loan CF. Matrix computations 3. JHU Press. 2012.

[50] Tzourio-Mazoyer N, Landeau B, Papathanassiou D et al. Automated Anatomical Labeling of activations in SPM using a Macroscopic Anatomical Parcellation of the MNI MRI single-subject brain. NeuroImage. 2002; 15 (1): 273-289. 


\section{$\underline{\text { List of captions }}$}

\section{Figures}

Fig. 1: Schematic representation of the electrodes positions in the (a) 19-Electrodes (b) 33-Electrodes (c) 71 Electrodes EEG headset configurations.

Fig. 2: Lateral Surface of the Brodmann map. (1)

Fig. 3: Brodmann area 8 (white dotted) shown over the original subdivision of the cortex into 6203 dipoles.

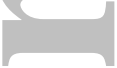

Fig. 4: Brodmann area 5 (white dotted) shown over the original subdivision of the cortex into 6203 dipoles.

Fig. 5: Influence of the number of simultaneously active areas on the performance of the reconstruction.

Fig. 6: Localization error analysis for unsuccessful cases, where "success" meant that all the activated

Functional Zones were exactly located in the reconstructed signal.

Fig. 7: Success rate for different levels of SNR, in the case of single Functional Zone.

Fig. 8: Singular values of lead field matrix for Brodmann areas with, (a) 19 electrodes, (b) 33 electrodes, (c) 71

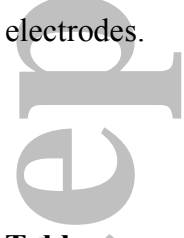

Tables

Table 1. Success rate and the localization error analysis for unsuccessful cases for one Active Zone.<smiles>[CH]1CCC1</smiles>

Table 2: Success rate as a function of the activated percentage of a Functional Zone (for 33 Electrodes Setup).

Table 3: Success rate with respect to the percentage of the active area (for 33 Electrodes Setup). The results shown here are averages over 10 runs.

Table 4: Success rate and the localization error analysis for one active dipole. 
Table 1. Success rate and the localization error analysis for unsuccessful cases for one Active Zone.

\begin{tabular}{|c|c|c|c|c|c|c|}
\hline & \multicolumn{3}{|c|}{ TMSBL } & \multicolumn{3}{|c|}{ sLORETA } \\
\hline $\begin{array}{l}\text { Number of } \\
\text { Electrodes }\end{array}$ & $\begin{array}{c}\text { Success } \\
\text { rate }\end{array}$ & $\begin{array}{c}\text { Mean error } \\
\text { distance }(\mathrm{mm})\end{array}$ & $\begin{array}{c}\text { Standard } \\
\text { deviation } \\
(\mathrm{mm})\end{array}$ & Success rate & $\begin{array}{c}\text { Mean error } \\
\text { distance } \\
\text { (mm) }\end{array}$ & $\begin{array}{l}\text { Standard } \\
\text { deviation } \\
\text { (mm) }\end{array}$ \\
\hline 19 & 0.86 & 20.62 & 11.96 & 0 & 41.00 & 5.91 \\
\hline & 0.98 & 14.94 & 10.27 & 0 & 30.93 & 6.92 \\
\hline 71 & 1.00 & 0 & 0 & 0.01 & 24.19 & 6.53 \\
\hline
\end{tabular}

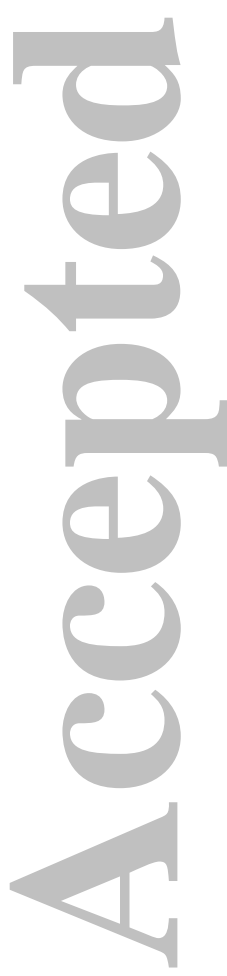


Table 2. Success rate as a function of the activated percentage of a Functional Zone (for 33 Electrodes Setup).

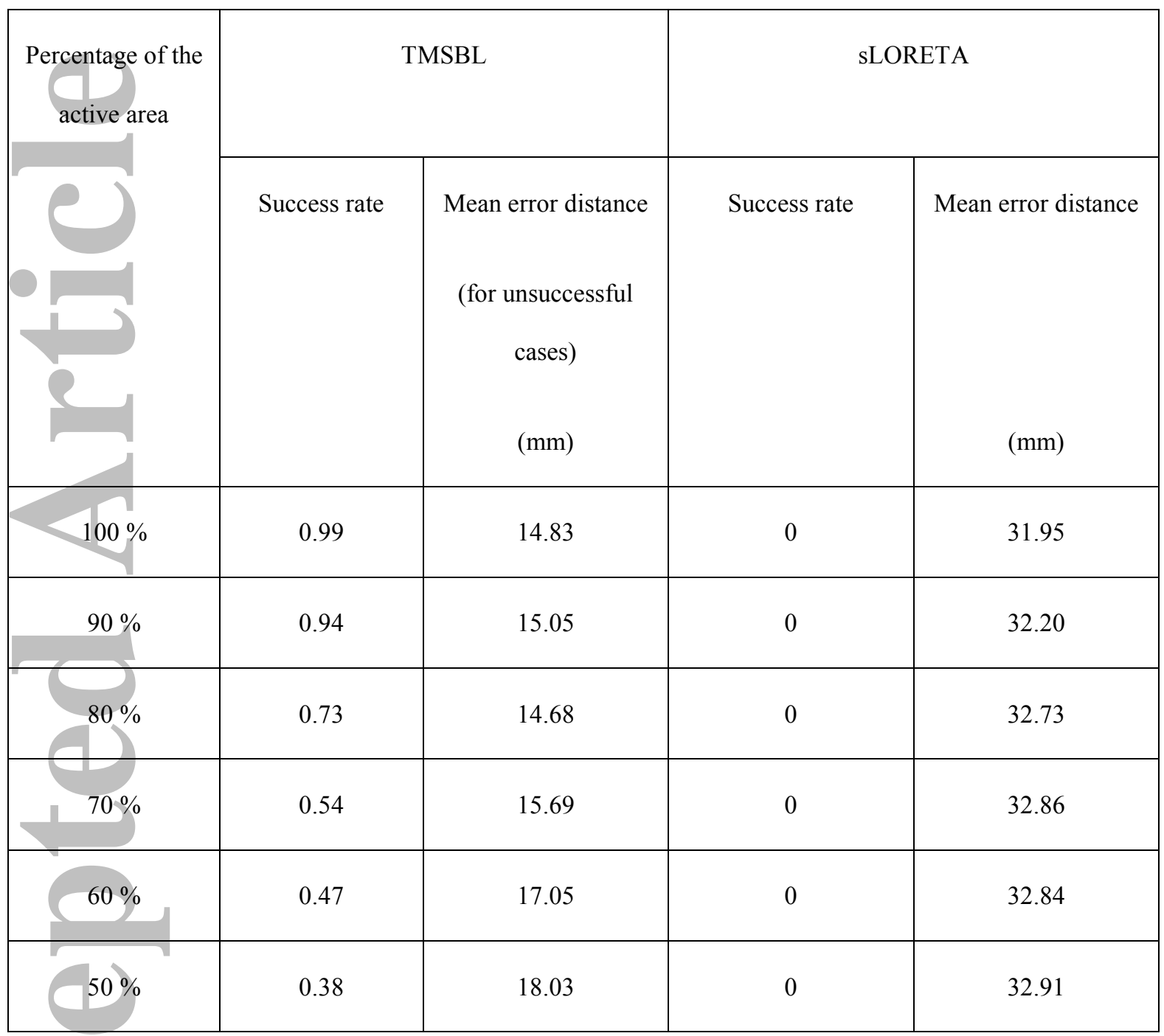


Table 3. Success rate with respect to the percentage of the active area (for 33 Electrodes Setup). The results shown here are averages over 10 runs.

\begin{tabular}{|c|c|c|c|c|}
\hline \multirow{2}{*}{$\begin{array}{l}\text { Percentage of the } \\
\text { active area }\end{array}$} & \multicolumn{2}{|c|}{ TMSBL } & \multicolumn{2}{|c|}{ sLORETA } \\
\hline & Success rate & $\begin{array}{c}\text { Mean Euclidian distance } \\
\text { (for unsuccessful cases) } \\
(\mathrm{mm})\end{array}$ & Success rate & $\begin{array}{c}\text { Mean error distance } \\
\text { (mm) }\end{array}$ \\
\hline $100 \%$ & 0.99 & 16.05 & 1.00 & 0 \\
\hline & 0.98 & 17.03 & 0.99 & 6.67 \\
\hline $80 \%$ & 0.95 & 16.87 & 0.98 & 12.91 \\
\hline $70 \%$ & 0.89 & 17.56 & 0.97 & 14.16 \\
\hline $60 \%$ & 0.84 & 17.89 & 0.87 & 17.98 \\
\hline $50 \%$ & 0.78 & 21.34 & 0.83 & 18.41 \\
\hline
\end{tabular}


Table 4. Success rate and the localization error analysis for one active dipole.

\begin{tabular}{|c|c|c|c|}
\hline Number of Electrodes & Success rate & $\begin{array}{r}\text { Mean error distance } \\
\text { (for unsuccessful cases) }\end{array}$ & $\begin{array}{c}\text { Standard deviation } \\
\text { (for unsuccessful cases) } \\
(\mathrm{mm})\end{array}$ \\
\hline 33 & 0 & $(\mathrm{~mm})$ & 5.66 \\
\hline 71 & 0 & 55.32 & 4.25 \\
\hline 7 & 1 & 42.20 & 0.00 \\
\hline
\end{tabular}

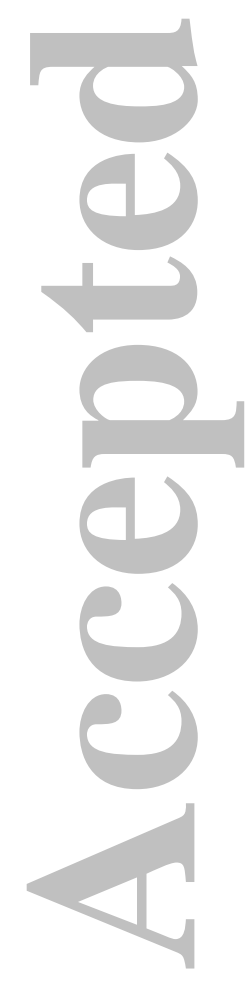




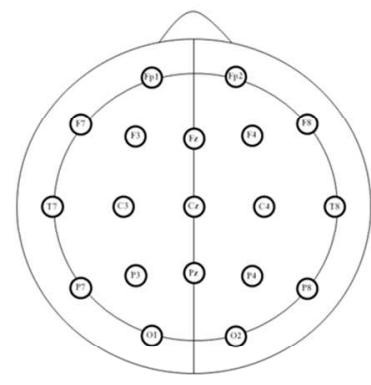

(a)

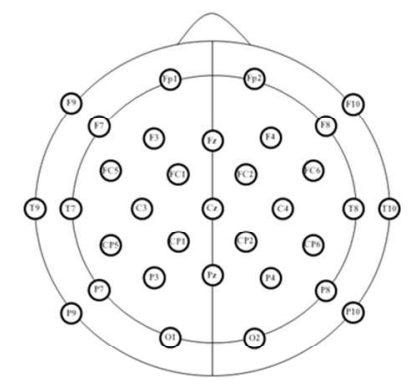

(b)

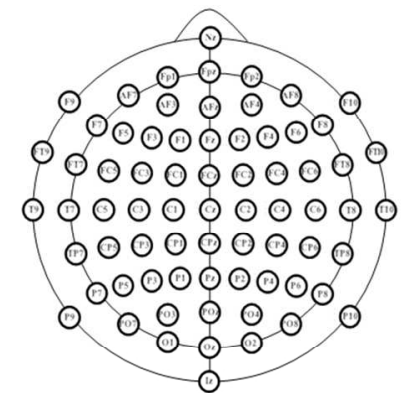

(c)

Figure 1: Schematic representation of the electrodes positions in the (a) 19-Electrodes (b) 33-Electrodes (c) 71-Electrodes EEG headset configurations.

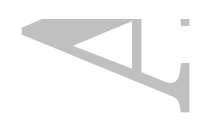

$153 \times 54 \mathrm{~mm}(300 \times 300$ DPI $)$

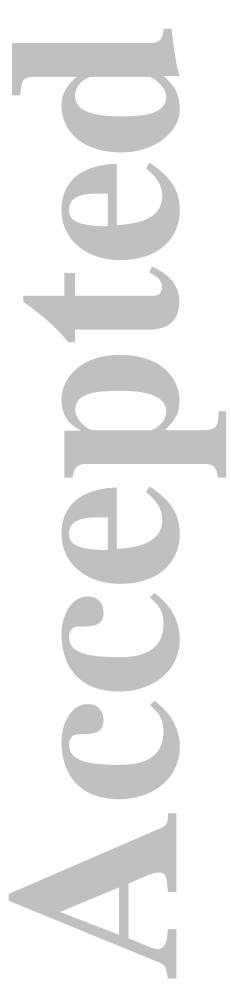

John Wiley \& Sons

This article is protected by copyright. All rights reserved. 


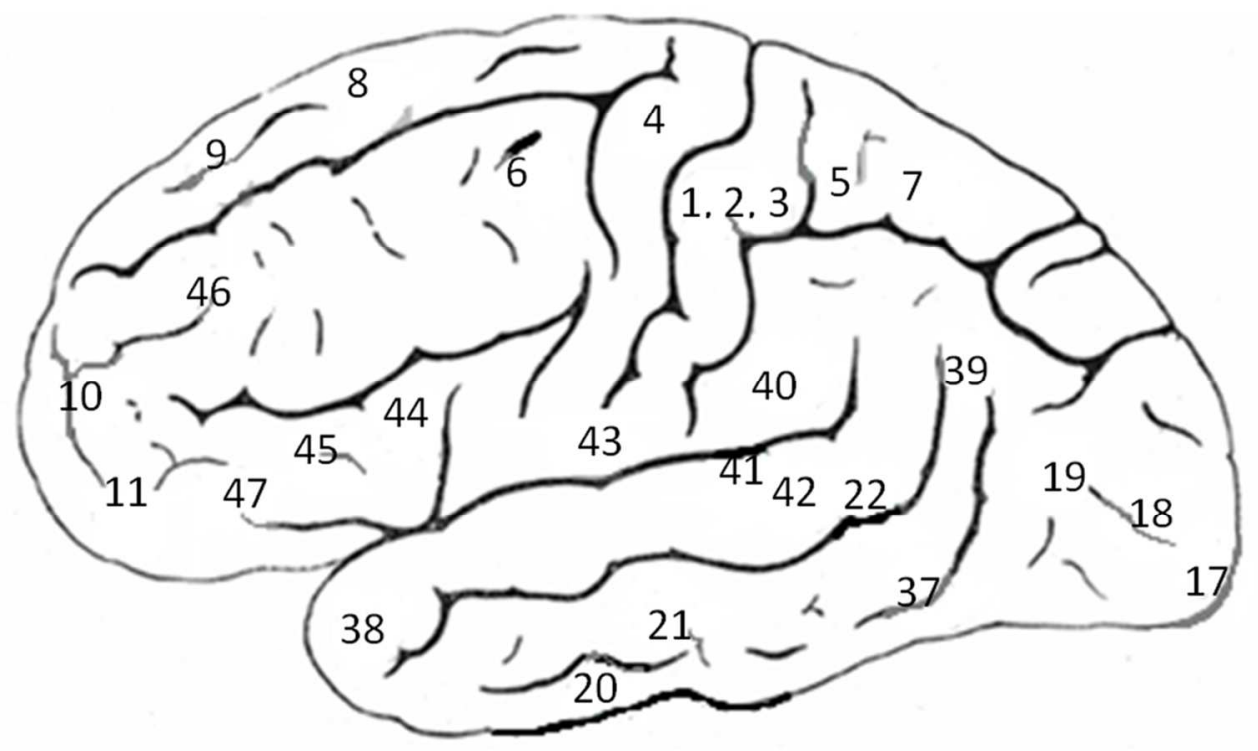

Figure 2: Lateral Surface of the Brodmann map. $104 \times 64 \mathrm{~mm}(300 \times 300 \mathrm{DPI})$

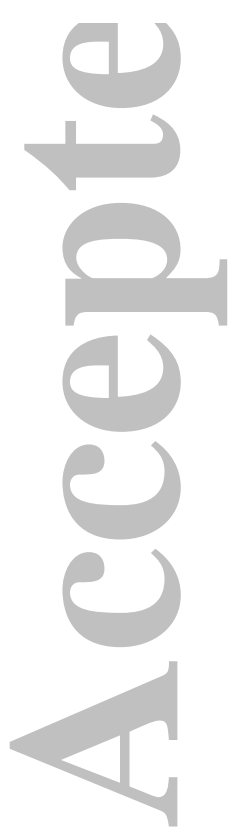




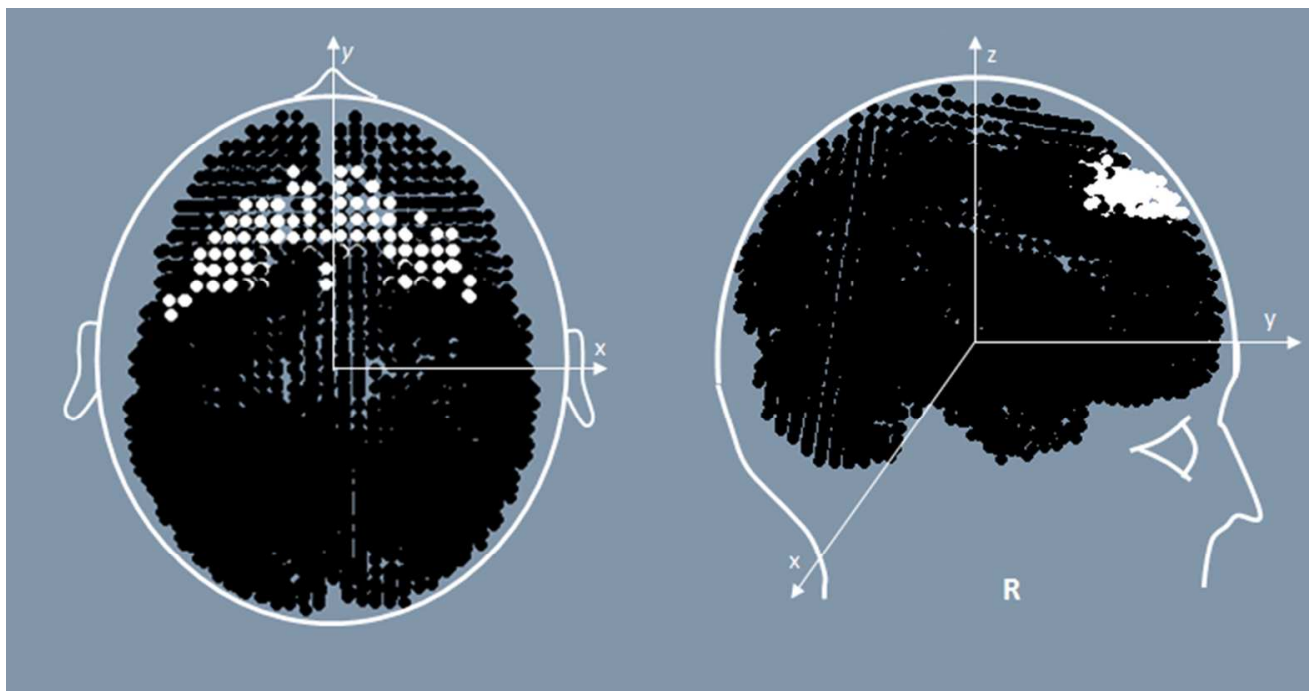

Figure 3: Brodmann area 8 (white dotted) shown over the original subdivision of the cortex into 6203 dipoles.

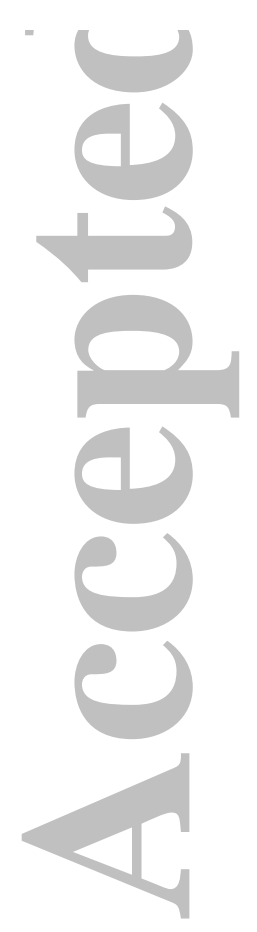

$116 \times 61 \mathrm{~mm}(300 \times 300 \mathrm{DPI})$ 


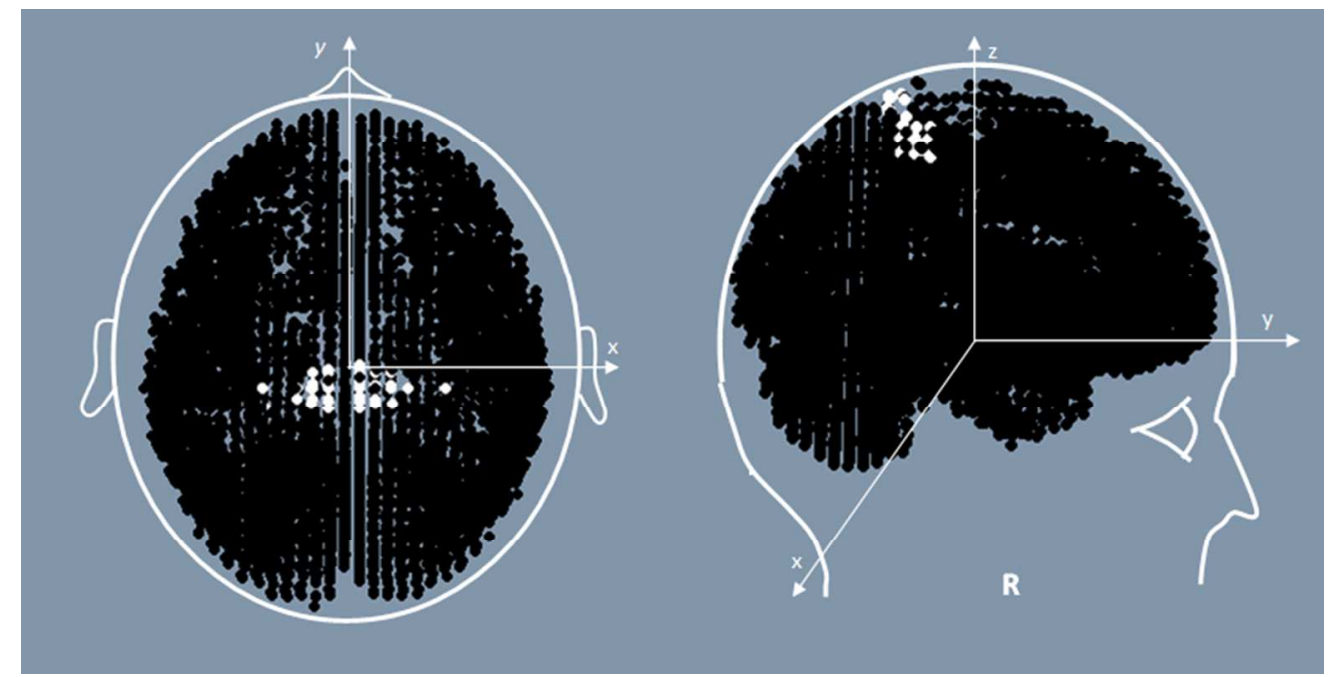

Figure 4: Brodmann area 5 (white dotted) shown over the original subdivision of the cortex into 6203 dipoles.

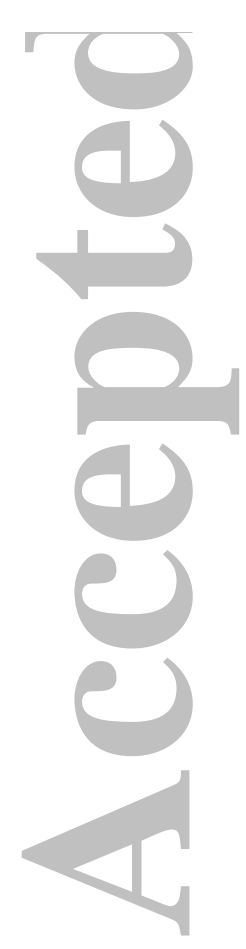

$120 \times 61 \mathrm{~mm}(300 \times 300 \mathrm{DPI})$ 


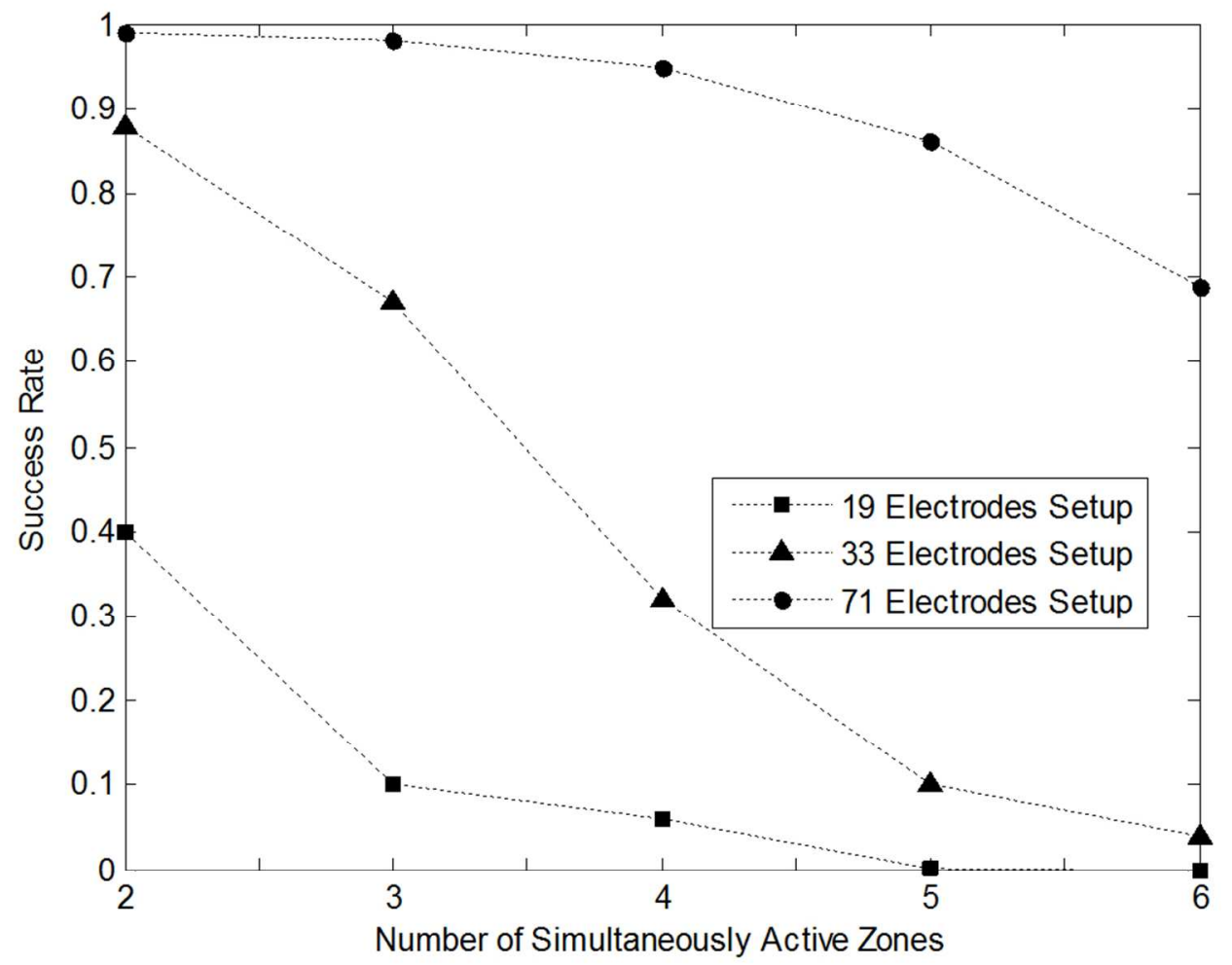

Figure 5: Influence of the number of simultaneously active areas on the performance of the reconstruction.

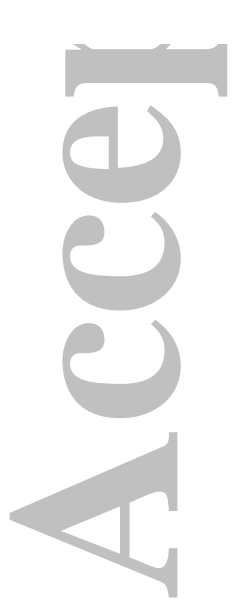

$90 \times 72 \mathrm{~mm}(300 \times 300 \mathrm{DPI})$

John Wiley \& Sons

This article is protected by copyright. All rights reserved. 


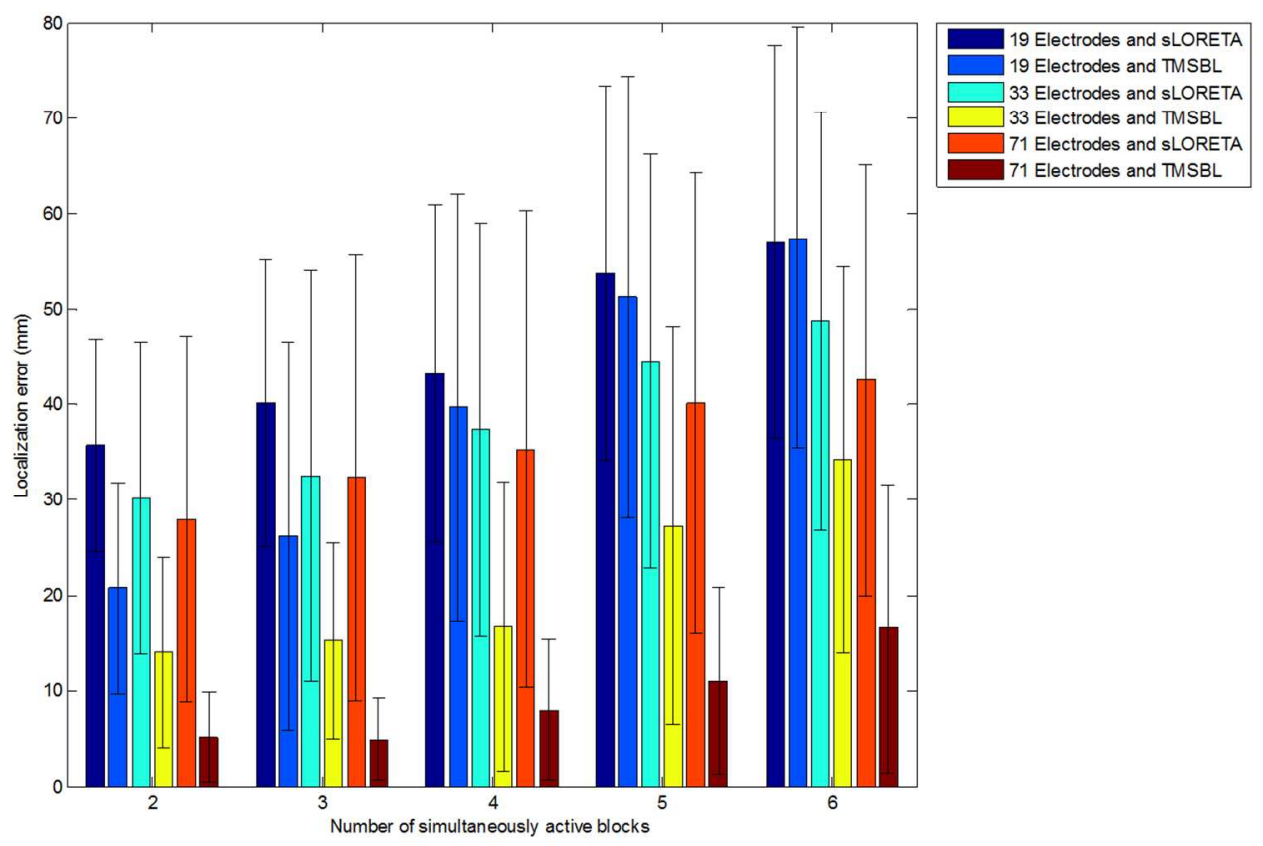

Figure 6: Localization error analysis for unsuccessful cases, where "success" meant that all the activated Functional Zones were exactly located in the reconstructed signal.

$113 \times 77 \mathrm{~mm}(300 \times 300 \mathrm{DPI})$

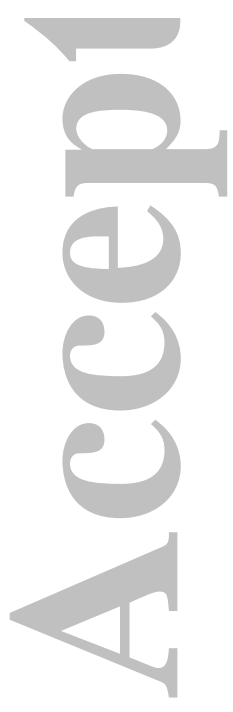




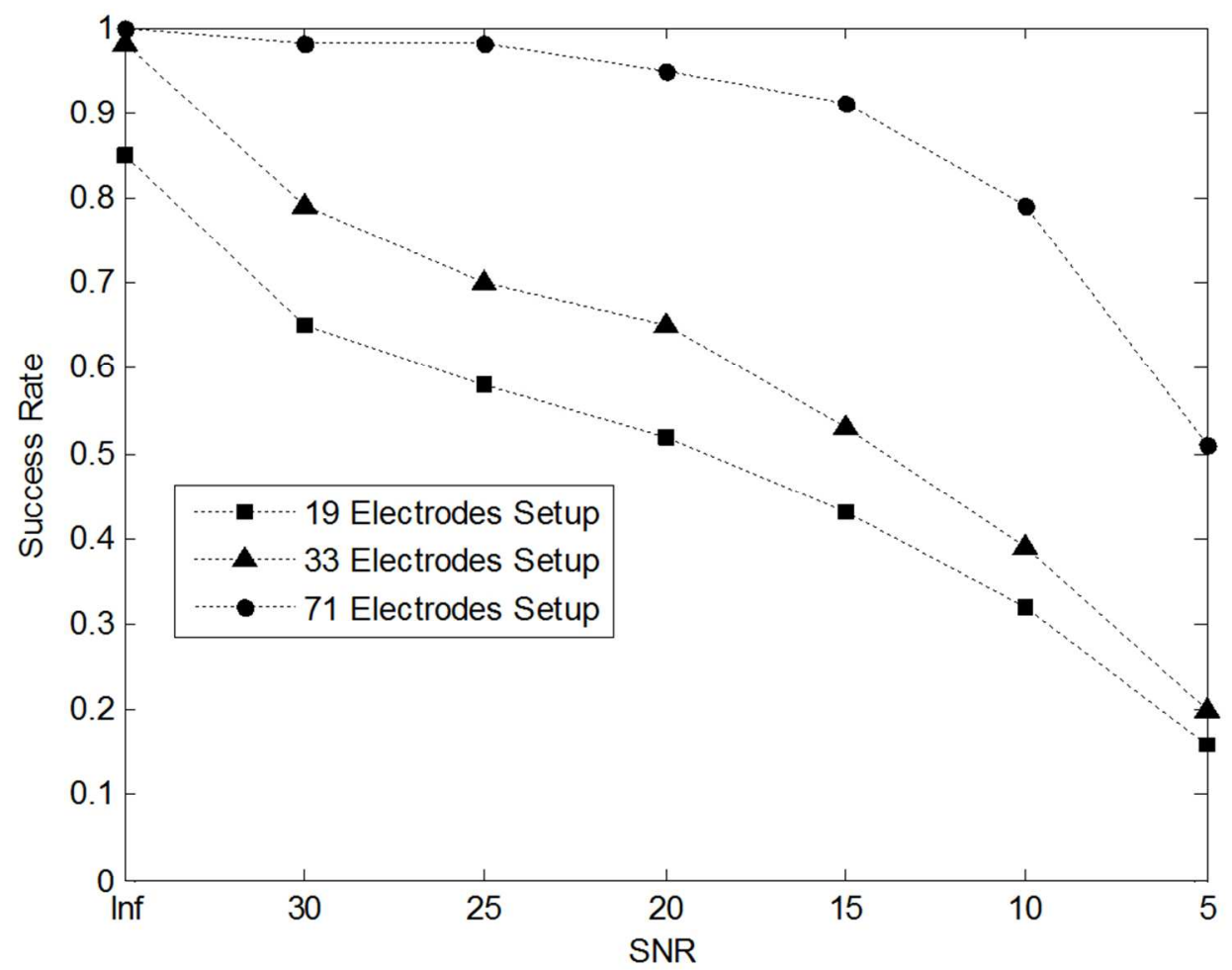

Figure 7: Success rate for different levels of SNR, in the case of single Functional Zone.

$92 \times 73 \mathrm{~mm}(300 \times 300 \mathrm{DPI})$

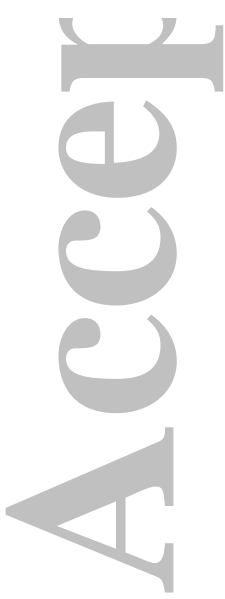

John Wiley \& Sons

This article is protected by copyright. All rights reserved. 


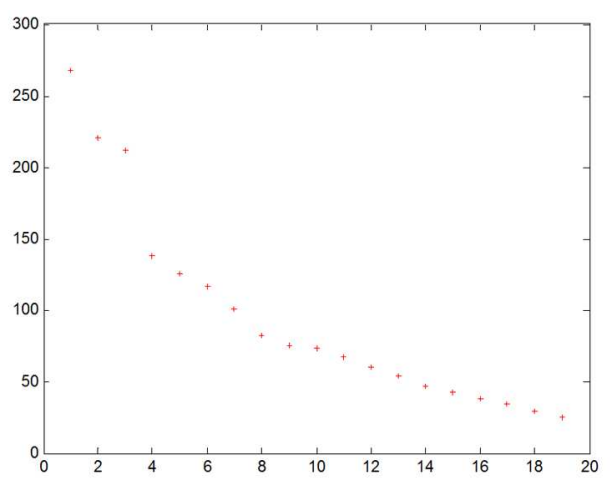

(a)

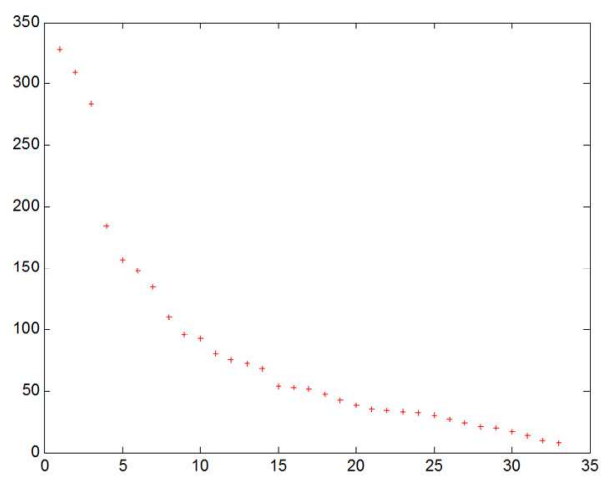

(b)

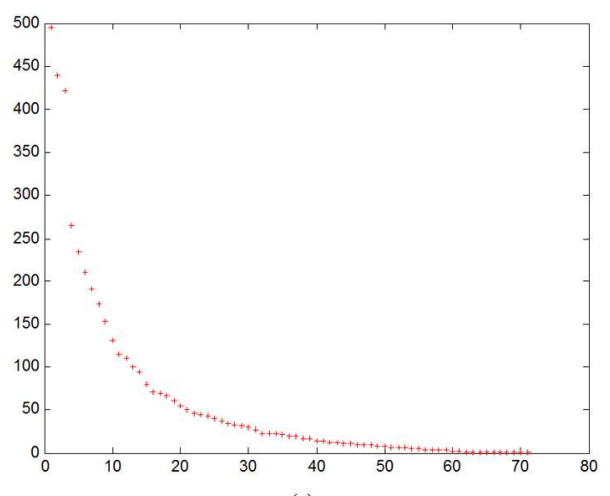

(c)

Figure 8: Singular values of lead field matrix for Brodmann areas with, (a) 19 electrodes, (b) 33 electrodes, (c) 71 electrodes.

$73 \times 180 \mathrm{~mm}(300 \times 300$ DPI $)$ 


\section{University Library}

\section{- M M N E R VA A gateway to Melbourne's research publications}

Minerva Access is the Institutional Repository of The University of Melbourne

Author/s:

Saha, S;Nesterets, Y;Rana, R;Tahtali, M;de Hoog, F;Gureyev, T

Title:

EEG source localization using a sparsity prior based on Brodmann areas

Date:

2017-12-01

Citation:

Saha, S., Nesterets, Y., Rana, R., Tahtali, M., de Hoog, F. \& Gureyev, T. (2017). EEG source localization using a sparsity prior based on Brodmann areas. INTERNATIONAL JOURNAL OF IMAGING SYSTEMS AND TECHNOLOGY, 27 (4), pp.333-344. https://doi.org/10.1002/ ima.22236.

Persistent Link:

http://hdl.handle.net/11343/293886 